\title{
A One-Pot Process for the Enantioselective Synthesis of Amines via Reductive Amination under Transfer Hydrogenation Conditions.
}

\author{
Glynn Williams, ${ }^{\dagger}$ Richard Pike, ${ }^{\dagger}$ Charles Wade ${ }^{\ddagger}$ and Martin Wills. ${ }^{\dagger *}$ \\ Department of Chemistry, University of Warwick, Coventry, CV4 7AL, UK. Process Chemistry Department, \\ GlaxoSmithKline Pharmaceuticals Ltd, Gunnels Wood Road, Stevenage, Herts, SG1 2NY. \\ m.wills@warwick.ac.uk
}

Supporting data; Experimental procedures and spectroscopic data for all new compounds.

\section{Contents}

General

General procedure for preparation of $t$-Boc protected lactams

Compound (6)

General procedure for the preparation of t-Boc-amino-ketones

(5-oxo-5-phenyl-pentyl)-carbamic acid tert-butyl ester

(5-oxo-5-cyclohexyl-pentyl)-carbamic acid tert-butyl ester

(5-oxo-5-(2-methoxyphenyl)-pentyl)-carbamic acid tert-butyl ester

(5-oxo-5-(3-methoxyphenyl)-pentyl)-carbamic acid tert-butyl ester

(5-oxo-5-(4-methoxyphenyl)-pentyl)-carbamic acid tert-butyl ester

(5-oxo-5-phenyl-butyl)-carbamic acid tert-butyl ester (14)

(5-oxo-5-phenyl-hexyl)-carbamic acid tert-butyl ester (13)

(5-oxo-5-(2-thiophene)-pentyl)-carbamic acid tert-butyl ester

(5-oxo-5-(3-trifluoromethylphenyl)-pentyl)-carbamic acid tert-butyl ester

(5-oxo-5-(4-trifluoromethylphenyl)-pentyl)-carbamic acid tert-butyl ester

General procedure for the racemic preparation of amines

( \pm )-2-phenyl-piperidine (7)

$( \pm)$-2-cyclohexyl-piperidine

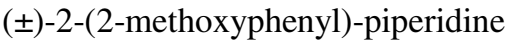

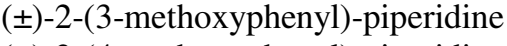

(士)-2-(4-methoxyphenyl)-piperidine

(士)-2-phenyl-cycloheptylamine

( \pm )-2-Thiophenyl-2-yl-piperidine

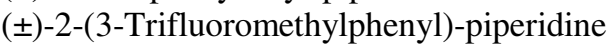

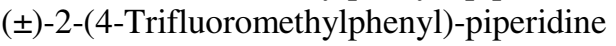

Preparation (One-pot process) of piperidines.

Asymmetric reduction of $\mathbf{9}$ to $\mathbf{1 0}$

$\begin{array}{lr}\text { Preparation of } \mathbf{1 1} \text { from lactam 6,7-dimethoxy-3,4-dihydro-2H-isoquinolin-1-one. } & 16 \\ \text { Preparation of } \mathbf{1 1} & 17\end{array}$

General procedure for determination of enantiomeric excess. $\quad 18$

References. 


\section{General}

All reactions, unless otherwise stated, were run under an atmosphere of nitrogen at ambient temperature $\left(18-22^{\circ} \mathrm{C}\right) .0^{\circ} \mathrm{C}$ refers to an ice/water slush bath and $-78^{\circ} \mathrm{C}$ refers to a dry ice-acetone bath. Heated experiments were conducted using thermostatically controlled oil baths. Reactions were monitored by TLC using aluminium backed silica gel 60 (F254) plates, visualised using UV 254 and 2-4-dinitrophenylhydrazine, ninhydrin and potassium permanganate dips as appropriate. Flash column chromatography was carried out routinely using $60 \AA$ silica gel (Merck). Reagents were used as received from commercial sources apart from the following exceptions, THF was distilled from sodium benzophenone ketyl, triethylamine was distilled from calcium hydride and formic acid was distilled from phthalic anhydride. NMR spectra were recorded on a Bruker DPX (300 or $400 \mathrm{MHz}$ ) spectrometer. Chemical shifts are reported in $\delta$ units, parts per million downfield from TMS. Coupling constants $(J)$ are measured in hertz. IR spectra were recorded on a Perkin-Elmer spectrum One FT-IR Golden Gate. Mass spectra were recorded on a 7070E VG mass spectrometer. Melting points were recorded on a Stuart Scientific SMP 1 instrument and are uncorrected.

\section{General procedure for preparation of $t$-Boc protected lactams. ${ }^{1}$}

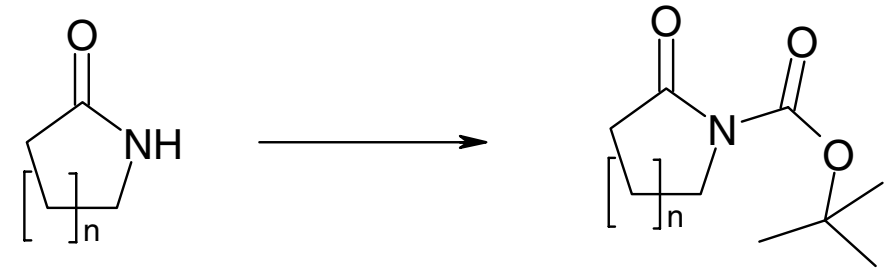

To a stirred solution of lactam $(0.1 \mathrm{~mol})$ in anhydrous THF $(200 \mathrm{~mL})$ at $-78^{\circ} \mathrm{C}$ was added drop wise n$\operatorname{BuLi}\left(0.1 \mathrm{~mol}, 64 \mathrm{~mL}, 2.5 \mathrm{M}\right.$ sol.) maintaining the temperature below $-72^{\circ} \mathrm{C}$. The mixture was allowed to stir at $-78^{\circ} \mathrm{C}$ for $1 \mathrm{~h}$ before a solution of di-tert-butyldicarbonate $(0.1 \mathrm{~mol})$ in THF $(25 \mathrm{~mL})$ was added drop wise over 45 minutes. The reaction was allowed to stir for a further $2 \mathrm{~h}$ and then warmed to rt. Sat $\mathrm{NH}_{4} \mathrm{Cl}(100 \mathrm{~mL})$ and water $(50 \mathrm{~mL})$ was added and the organic layer was separated. The aqueous phase was extracted with diethyl ether $(3 \mathrm{x} 100 \mathrm{~mL})$. The combined organic phases were dried $\left(\mathrm{MgSO}_{4}\right)$ and the solvent removed under reduced pressure. The residue was purified by flash column chromatography ( $20 \% \mathrm{v} / \mathrm{v}$ ethyl acetate-hexanes) to afford the Boc-protected lactams. 


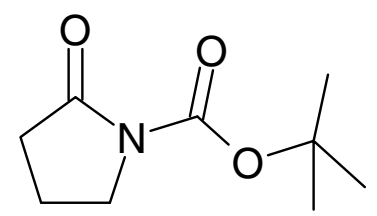

Colourless oil, (14.2 g, $77 \%$ ) ; $v_{\max }$ (neat) $/ \mathrm{cm}^{-1} 3399,2978,2361,1780,1748,1706,1365,1299$, $1147 ; \delta_{\mathrm{H}}\left(300.13 \mathrm{MHz}, \mathrm{CDCl}_{3}\right) 3.75\left(2 \mathrm{H}, \mathrm{t}, J 7.01, \mathrm{NCH}_{2}\right), 2.51\left(2 \mathrm{H}, \mathrm{t}, J\right.$ 8.53, $\left.\mathrm{COCH}_{2}\right), 1.96-2.03$ $\left(2 \mathrm{H}, \mathrm{m}, \mathrm{CH}_{2} \mathrm{CH}_{2} \mathrm{CH}_{2}\right), 1.53(9 \mathrm{H}, \mathrm{s}, t-\mathrm{Bu}) ; \delta_{\mathrm{C}}\left(75.5 \mathrm{MHz}, \mathrm{CDCl}_{3}\right) 174.6\left(\mathrm{C}_{\mathrm{q}}\right), 150.4\left(\mathrm{C}_{\mathrm{q}}\right), 82.8\left(\mathrm{C}_{\mathrm{q}}\right)$, $46.8\left(\mathrm{CH}_{2}\right), 33.2\left(\mathrm{CH}_{2}\right), 28.3\left(\mathrm{CH}_{3}\right), 17.7\left(\mathrm{CH}_{2}\right) ; \mathrm{MS} \mathrm{m} / z$ (EI) 112([M+H-Ot-Bu] $\left.]^{+}, 6\right), 86$ (37), 57 (100) ; Anal. calc. for $\mathrm{C}_{9} \mathrm{H}_{15} \mathrm{NO}_{3}: \mathrm{C}, 58.36 ; \mathrm{H}, 8.16$; N, 7.56. Found: C, 58.08; H, 8.20; N, 7.30. ${ }^{1}$

\section{Compound (6)}<smiles>CC(C)(C)OC(=O)N1CCCCC1=O</smiles>

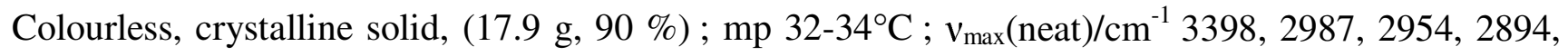
$2360,1701,1285,1246,1155,1133 ; \delta_{\mathrm{H}}\left(300.13 \mathrm{MHz}, \mathrm{CDCl}_{3}\right) 3.65\left(2 \mathrm{H}, \mathrm{m}, \mathrm{NCH}_{2}\right), 2.50(2 \mathrm{H}, \mathrm{m}$, $\left.\mathrm{COCH}_{2}\right), 1.82\left(4 \mathrm{~h}, \mathrm{~m}, \mathrm{NCH}_{2} \mathrm{CH}_{2} \mathrm{CH}_{2}\right), 1.52(9 \mathrm{H}, \mathrm{s}, t-\mathrm{Bu}) ; \delta_{\mathrm{C}}\left(75.5 \mathrm{MHz}, \mathrm{CDCl}_{3}\right) 171.6\left(\mathrm{C}_{\mathrm{q}}\right), 152.9$ $\left(\mathrm{C}_{\mathrm{q}}\right)$, 82.8, $\left(\mathrm{C}_{\mathrm{q}}\right), 46.4\left(\mathrm{CH}_{2}\right), 34.9\left(\mathrm{CH}_{2}\right), 28.3\left(\mathrm{CH}_{3}\right), 23.0\left(\mathrm{CH}_{2}\right), 20.8\left(\mathrm{CH}_{2}\right) ; \mathrm{MS} \mathrm{m} / z$ (EI) $144([\mathrm{M}-t-$ $\left.\mathrm{Bu}]^{+}, 35\right), 100$ (17), 83 (100); Anal. calc. for $\mathrm{C}_{10} \mathrm{H}_{17} \mathrm{NO}_{3}$ : C, 60.28; H, 8.60; N, 7.03. Found: C, 60.30; H, 8.60; N, 6.98. ${ }^{1}$

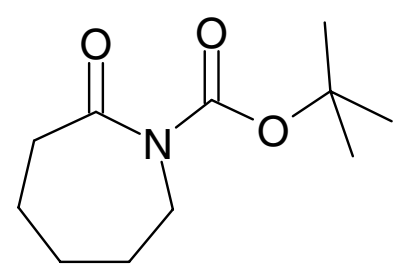

Colourless oil, (13.2 g, $62 \%) ; v_{\max }\left(\right.$ neat) $/ \mathrm{cm}^{-1} 2977,2930,2358,1766,1705,1142 ; \delta_{\mathrm{H}}(300.13 \mathrm{MHz}$, $\left.\mathrm{CDCl}_{3}\right) \quad 3.72-3.80\left(2 \mathrm{H}, \quad \mathrm{m}, \quad \mathrm{NCH}_{2}\right), \quad 2.62-2.70 \quad\left(2 \mathrm{H}, \quad \mathrm{m}, \quad \mathrm{COCH}_{2}\right), \quad 1.70-1.85 \quad(6 \mathrm{H}, \quad \mathrm{m}$, $\left.\mathrm{COCH}_{2} \mathrm{CH}_{2} \mathrm{CH}_{2} \mathrm{CH}_{2}\right), 1.52(9 \mathrm{H}, \mathrm{s}, t-\mathrm{Bu}) ; \delta_{\mathrm{C}}\left(75.5 \mathrm{MHz}, \mathrm{CDCl}_{3}\right) 176.0\left(\mathrm{C}_{\mathrm{q}}\right), 153.2\left(\mathrm{C}_{\mathrm{q}}\right), 83.0\left(\mathrm{C}_{\mathrm{q}}\right)$, $46.5\left(\mathrm{CH}_{2}\right), 39.8\left(\mathrm{CH}_{2}\right), 29.5\left(\mathrm{CH}_{2}\right), 29.0\left(\mathrm{CH}_{2}\right), 28.3\left(\mathrm{CH}_{3}\right), 23.9\left(\mathrm{CH}_{2}\right) ; \mathrm{MS} \mathrm{m} / \mathrm{z}$ (EI) 214 ([M+H] $\left.]^{+}, 45\right), 213$ (23), 209 (100), 158 (99), 140 (17), 85 (37); Anal. calc. for $\mathrm{C}_{11} \mathrm{H}_{19} \mathrm{NO}_{3}: \mathrm{C}, 61.95$; H, 8.98; N, 6.57. Found: C, 62.13; H, 9.11; N, 6.46. ${ }^{1}$ 


\section{General procedure for the preparation of t-Boc-amino-ketones}
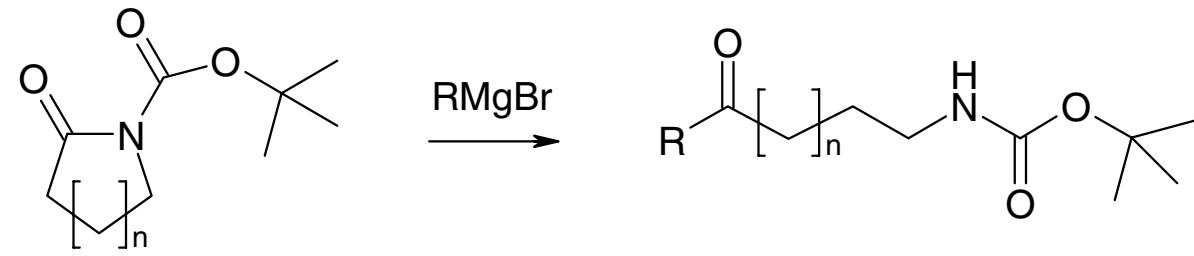

The Grignard reagent was prepared by the following procedure unless it was commercially available. To a dry 2 necked flask was added Mg $(0.01 \mathrm{~mol})$, dry THF $(5 \mathrm{~mL})$ and the aryl bromine $(0.006 \mathrm{~mol})$ with stirring under $\mathrm{N}_{2}$. Iodine crystals (catalytic) were added and the mixture was heated gently until it maintained its own reflux. When reflux had subsided external heating was applied to maintain reflux for a further 1 hour. The $t$-Boc-lactam $(0.005 \mathrm{~mol})$ was added to a dry 3 necked round bottomed flask with a thermometer. Dry THF $(20 \mathrm{~mL})$ was added with stirring under $\mathrm{N}_{2}$ and the solution was cooled to $-78^{\circ} \mathrm{C}$. The Grignard reagent $(0.006 \mathrm{~mol})$ was added to the t-Boc-lactam over 1 hour, maintaining the internal temperature below $-70^{\circ} \mathrm{C}$. The solution was warmed to room temperature and $2 \mathrm{M} \mathrm{HCl}$ was added until the $\mathrm{pH}$ was $1-3$. The aqueous layer was washed $3 \times 50 \mathrm{~mL}$ with DCM and the organic layers combined, dried over $\mathrm{Na}_{2} \mathrm{SO}_{4}$, filtered and concentrated in vacuo. The product was purified using flash column chromatography (F.C.C.), (15-20\% v/v ethyl acetate / hexane) or recrystallisation from pet. ether (40-60).

\section{(5-oxo-5-phenyl-pentyl)-carbamic acid tert-butyl ester. ${ }^{1}$}

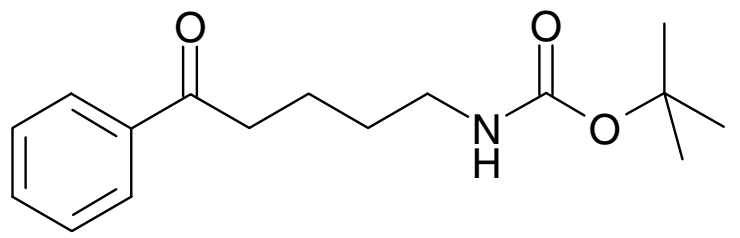

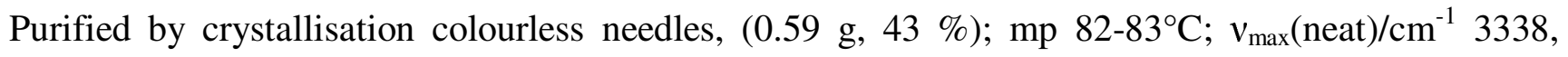
2973, 2945, 2927, 2361, 2342, 1679, 1533, 1281, 1248, 1169, 1133, 730, 687, 653; $\delta_{\mathrm{H}}(300.13 \mathrm{MHz}$, $\left.\mathrm{CDCl}_{3}\right) 7.95(2 \mathrm{H}, \mathrm{d}, J$ 7.5, aryl H), $7.50(3 \mathrm{H}, \mathrm{m}$, aryl H), $4.60(1 \mathrm{H}, \mathrm{s}, \mathrm{NH}), 3.18(2 \mathrm{H}, \mathrm{q}, J 6.4$, $\left.\mathrm{NHCH}_{2}\right), 3.01\left(2 \mathrm{H}, \mathrm{t}, J\right.$ 6.8, $\left.\mathrm{COCH}_{2}\right), 1.78\left(2 \mathrm{H}, \mathrm{m}, \mathrm{NCH}_{2} \mathrm{CH}_{2}\right), 1.57\left(2 \mathrm{H}, \mathrm{m}, \mathrm{COCH}_{2} \mathrm{CH}_{2}\right), 1.44(9 \mathrm{H}$, s, $t$-Bu); $\delta_{\mathrm{C}}\left(75.5 \mathrm{MHz}, \mathrm{CDCl}_{3}\right) 155.8(\mathrm{C}), 136.7(\mathrm{C}), 128.4\left(\mathrm{CH}_{2}\right), 127.9\left(\mathrm{CH}_{2}\right), 78.9(\mathrm{C}), 40.0\left(\mathrm{CH}_{2}\right)$, $37.7\left(\mathrm{CH}_{2}\right), 29.3\left(\mathrm{CH}_{2}\right), 28.2\left(\mathrm{CH}_{2}\right), 21.0\left(\mathrm{CH}_{3}\right) ; \mathrm{MS} \mathrm{m} / z(\mathrm{EI}) 278\left([\mathrm{M}+\mathrm{H}]^{+}, 14\right), 176(100), 160$ (99), 105 (95), 77 (54); Anal. calc: $\mathrm{C}_{16} \mathrm{H}_{23} \mathrm{NO}_{3}: \mathrm{C}, 69.29$; H, 8.36; N, 5.05. Found: C, 69.34; H, 8.39; N, 5.00 . 
(5-oxo-5-cyclohexyl-pentyl)-carbamic acid tert-butyl ester<smiles>CC(C)(C)OC(=O)NCCCCC(=O)C1CCCCC1</smiles>

Purified by F.C.C., pale yellow crystalline solid, $(0.45 \mathrm{~g}, 32 \%)$; mp 32-35 ${ }^{\circ} \mathrm{C} ; v_{\max }(\mathrm{neat}) / \mathrm{cm}^{-1} 3366$, 2974, 2927, 2854, 2361, 2341, 1695, 1508, 1449, 1364, 1246, 1167; $\delta_{\mathrm{H}}\left(300.13 \mathrm{MHz}, \mathrm{CDCl}_{3}\right) 4.66$ $(1 \mathrm{H}, \mathrm{s}, \mathrm{NH}), 3.11\left(2 \mathrm{H}, \mathrm{q}, J 5.9, \mathrm{NHCH}_{2}\right), 2.46\left(2 \mathrm{H}, \mathrm{t}, J\right.$ 7.4, $\left.\mathrm{COCH}_{2}\right), 2.41(1 \mathrm{H}, \mathrm{m}, \mathrm{COCH}) 1.81(4 \mathrm{H}$, $\left.\mathrm{m}, \mathrm{COCH}_{2} \mathrm{CH}_{2} \mathrm{CH}_{2} \mathrm{CH}_{2} \mathrm{NH}\right), 1.58\left(4 \mathrm{H}, \mathrm{m}, \mathrm{CH}\left(\mathrm{CH}_{2}\right)_{2}\right), 1.41\left(9 \mathrm{H}, \mathrm{s}, \mathrm{OC}\left(\mathrm{CH}_{3}\right)_{3}\right), 1.35(6 \mathrm{H}, \mathrm{m}$, $\left.\mathrm{CH}\left(\mathrm{CH}_{2}\right)_{2}\left(\mathrm{CH}_{2}\right)_{3}\right) ; \delta_{\mathrm{C}}\left(75.5 \mathrm{MHz}, \mathrm{CDCl}_{3}\right) 156.4(\mathrm{C}), 82.5(\mathrm{C}), 79.4(\mathrm{C}), 51.2(\mathrm{CH}), 40.5\left(\mathrm{CH}_{2}\right), 40.3$ $\left(\mathrm{CH}_{2}\right), 29.9\left(\mathrm{CH}_{2}\right), 29.0,\left(\mathrm{CH}_{2}\right), 28.8\left(\mathrm{CH}_{3}\right), 26.2\left(\mathrm{CH}_{2}\right), 26.0\left(\mathrm{CH}_{2}\right), 21.0\left(\mathrm{CH}_{2}\right) ; \mathrm{MS} \mathrm{m} / z(\mathrm{EI}) 284$ ([M+H] $\left.]^{+}, 35\right), 228$ (55), 184 (100), 100 (75), 83 (84); Anal. calc: $\mathrm{C}_{16} \mathrm{H}_{29} \mathrm{NO}_{3}: \mathrm{C}, 67.81 ; \mathrm{H}, 10.31 ; \mathrm{N}$, 4.94. found $\mathrm{C}, 67.62 ; \mathrm{H}, 10.32 ; \mathrm{N} 4.96$.

(5-oxo-5-(2-methoxyphenyl)-pentyl)-carbamic acid tert-butyl ester<smiles>COc1ccccc1C(=O)CCCCNC(=O)OC(C)(C)C</smiles>

Purified by F.C.C. colourless oil, (0.64 g, $41 \%$ ); $v_{\max }\left(\right.$ neat) $/ \mathrm{cm}^{-1}$ 3358, 2973, 2931, 2867, 1673, 1596, $1509,1484,1456,1364,1242,1161,1022,981,755,735 ; \delta_{\mathrm{H}}\left(300.13 \mathrm{MHz}, \mathrm{CDCl}_{3}\right) 7.65(1 \mathrm{H}, \mathrm{dd}, J$ 7.6, 1.7, aryl H), $7.44\left(1 \mathrm{H}, \mathrm{m}\right.$, aryl H), $6.97\left(2 \mathrm{H}, \mathrm{m}\right.$, aryl H), $4.71(1 \mathrm{H}, \mathrm{s}, \mathrm{NH}), 3.86\left(3 \mathrm{H}, \mathrm{s}, \mathrm{OCH}_{3}\right)$, $3.14\left(2 \mathrm{H}, \mathrm{q}, J\right.$ 6.4, $\left.\mathrm{HNCH}_{2}\right), 2.98\left(2 \mathrm{H}, \mathrm{t}, J\right.$ 7.1, $\left.\mathrm{COCH}_{2}\right), 1.71\left(2 \mathrm{H}, \mathrm{m}, \mathrm{COCH}_{2} \mathrm{CH}_{2}\right), 1.54(2 \mathrm{H}, \mathrm{m}$, $\left.\mathrm{NHCH}_{2} \mathrm{CH}_{2}\right), 1.44(9 \mathrm{H}, \mathrm{s}, t-\mathrm{Bu}) ; \delta_{\mathrm{C}}\left(75.5 \mathrm{MHz}, \mathrm{CDCl}_{3}\right) 203.2(\mathrm{C}), 158.8(\mathrm{C}), 156.4(\mathrm{C}), 133.7(\mathrm{CH})$, $130.5(\mathrm{CH}), 128.8(\mathrm{C}), 121.0(\mathrm{CH}), 111.9(\mathrm{CH}), 79.3(\mathrm{C}), 55.9\left(\mathrm{CH}_{3}\right), 43.6\left(\mathrm{CH}_{2}\right), 40.7\left(\mathrm{CH}_{2}\right), 30.0$ $\left(\mathrm{CH}_{2}\right), 28.8\left(\mathrm{CH}_{3}\right), 21.8\left(\mathrm{CH}_{2}\right)$; MS m/z (CI) $325\left(\left[\mathrm{M}+\mathrm{NH}_{4}\right]^{+}, 11\right), 308$ (22), 269 (47), 252 (71), 234 (100), 190 (38); HRMS: $\mathrm{C}_{17} \mathrm{H}_{26} \mathrm{NO}_{4}(\mathrm{M}+\mathrm{H}$ \} calc 308.1856, found 308.1861.

\section{(5-0xo-5-(3-methoxyphenyl)-pentyl)-carbamic acid tert-butyl ester}




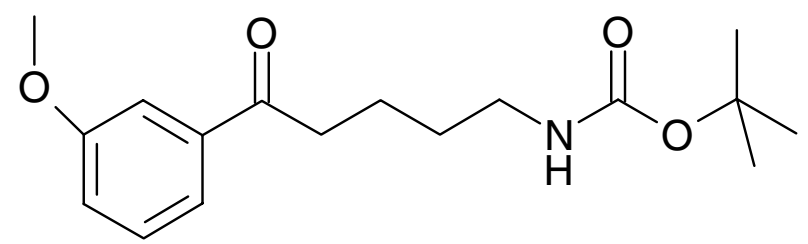

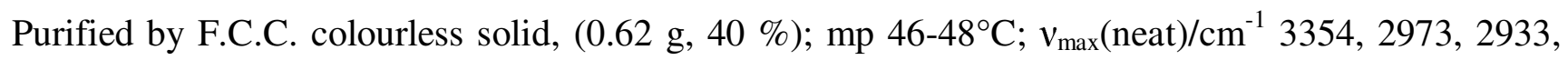
$1681,1514,1364,1250,1163,1040,994,781,735,685 ; \delta_{\mathrm{H}}\left(300.13 \mathrm{MHz}, \mathrm{CDCl}_{3}\right) 7.53(1 \mathrm{H}, \mathrm{m}$, aryl H), $7.48(1 \mathrm{H}, \mathrm{m}$, aryl H), $7.36(1 \mathrm{H}, \mathrm{t}, J$ 8.1, aryl H), $7.10(1 \mathrm{H}, \mathrm{m}, \operatorname{aryl~H}), 4.69(1 \mathrm{H}, \mathrm{s}, \mathrm{NH}), 3.84(3 \mathrm{H}$, s, $\left.\mathrm{OCH}_{3}\right), 3.16\left(2 \mathrm{H}, \mathrm{q}, J\right.$ 6.8, $\left.\mathrm{NHCH}_{2}\right), 2.99\left(2 \mathrm{H}, \mathrm{t}, J\right.$ 7.5, $\left.\mathrm{COCH}_{2}\right), 1.77(2 \mathrm{H}$, quin, $J$ 7.5, $\left.\mathrm{COCH}_{2} \mathrm{CH}_{2}\right), 1.57\left(2 \mathrm{H}, \mathrm{m}, \mathrm{NHCH}_{2} \mathrm{CH}_{2}\right), 1.44(9 \mathrm{H}, \mathrm{s}, t-\mathrm{Bu}) ; \delta_{\mathrm{C}}\left(75.5 \mathrm{MHz}, \mathrm{CDCl}_{3}\right) 200.4(\mathrm{C}), 160.2$ (C), $156.4(\mathrm{C}), 138.7(\mathrm{C}), 130.0(\mathrm{CH}), 121.1(\mathrm{CH}), 119.8(\mathrm{CH}), 112.6(\mathrm{CH}), 79.4(\mathrm{C}), 55.8\left(\mathrm{CH}_{3}\right)$, $40.6\left(\mathrm{CH}_{2}\right), 38.5\left(\mathrm{CH}_{2}\right), 30.0\left(\mathrm{CH}_{2}\right), 28.8\left(\mathrm{CH}_{3}\right), 21.7\left(\mathrm{CH}_{2}\right) ; \mathrm{MS} \mathrm{m} / \mathrm{z}(\mathrm{EI}) 308\left([\mathrm{M}+\mathrm{H}]^{+}, 100\right), 306$ (83), 300 (64), 298 (81); Anal. calc: $\mathrm{C}_{17} \mathrm{H}_{25} \mathrm{NO}_{4}$; C, 66.43; H, 8.20; N 4.56; found C, 66.36; H, 8.20; $\mathrm{N}, 4.43$.

\section{(5-0xo-5-(4-methoxyphenyl)-pentyl)-carbamic acid tert-butyl ester}<smiles>COc1ccc(C(=O)CCCCNC(=O)OC(C)(C)C)cc1</smiles>

Purified by F.C.C. colourless needles, (0.67 g, $43 \%$ ); mp 75-76 ${ }^{\circ}$; $v_{\max }($ neat $) / \mathrm{cm}^{-1} 3323,2982,2942$, $2866,2838,2359,2341,1685,1668,1604,1536,1276,1255,1159,1028,977,822,661 ; \delta_{\mathrm{H}}(300.13$ $\left.\mathrm{MHz}_{\mathrm{CDCl}}\right) 7.93(2 \mathrm{H}, \mathrm{d}, J$ 8.9, aryl H) $6.93(2 \mathrm{H}, \mathrm{d}, J$ 8.9, aryl H), $4.64(1 \mathrm{H}, \mathrm{s}, \mathrm{NH}), 3.85(3 \mathrm{H}, \mathrm{s}$, $\left.\mathrm{OCH}_{3}\right), 3.16\left(2 \mathrm{H}, \mathrm{q}, J\right.$ 6.6, $\left.\mathrm{NHCH}_{2}\right), 2.94\left(2 \mathrm{H}, \mathrm{t}, J\right.$ 7.1, $\left.\mathrm{COCH}_{2}\right), 1.76\left(2 \mathrm{H}\right.$, quin, $J$ 7.7, $\left.\mathrm{COCH}_{2} \mathrm{CH}_{2}\right)$, $1.57\left(2 \mathrm{H}, \mathrm{m}, \mathrm{NHCH}_{2} \mathrm{CH}_{2}\right), 1.44(9 \mathrm{H}, \mathrm{s}, t-\mathrm{Bu}) ; \delta_{\mathrm{C}}\left(75.5 \mathrm{MHz}, \mathrm{CDCl}_{3}\right) 199.3(\mathrm{C}), 163.8(\mathrm{C}), 156.4(\mathrm{C})$, $130.7(\mathrm{CH}), 130.4(\mathrm{C}), 114.1(\mathrm{CH}), 79.4(\mathrm{C}), 55.8\left(\mathrm{CH}_{3}\right), 40.6\left(\mathrm{CH}_{2}\right), 38.0\left(\mathrm{CH}_{2}\right), 30.0\left(\mathrm{CH}_{2}\right), 28.8$ $\left(\mathrm{CH}_{3}\right), 21.8\left(\mathrm{CH}_{2}\right)$; MS m/z (EI), $307\left([\mathrm{M}]^{+}, 30\right), 189$ (81), 135 (100), 121 (43), 77 (30); HRMS: $\mathrm{C}_{17} \mathrm{H}_{25} \mathrm{NO}_{4}$ calc 307.1784, found 307.1798; Anal. calc: $\mathrm{C}_{17} \mathrm{H}_{25} \mathrm{NO}_{4} \mathrm{C} 66.43, \mathrm{H} \mathrm{8.20,} \mathrm{N} \mathrm{4.56,} \mathrm{found} \mathrm{C}$ $66.29, \mathrm{H} 8.10, \mathrm{~N} 4.38$.

\section{(5-oxo-5-phenyl-butyl)-carbamic acid tert-butyl ester (14)}


<smiles>CC(C)(C)OC(=O)NCCCC(=O)c1ccccc1</smiles>

Purified by crystallisation as colourless needles, $(0.84 \mathrm{~g}, 64 \%) ; \mathrm{mp} 95-96^{\circ} \mathrm{C} ; v_{\max }($ neat $) / \mathrm{cm}^{-1} 3722$, 3365, 2988, 2934, 2359, 1679, 1516, 1245, 1155; $\delta_{\mathrm{H}}\left(300.13 \mathrm{MHz}, \mathrm{CDCl}_{3}\right)$ 7.94-7.98 (2H, m, aryl H), 7.43-7.59 (3H, m, aryl H), $4.68(1 \mathrm{H}, \mathrm{bs}, \mathrm{NH}), 3.19-3.26\left(2 \mathrm{H}, \mathrm{m}, \mathrm{NCH}_{2}\right), 3.03\left(2 \mathrm{H}, \mathrm{t}, J\right.$ 7.2, $\left.\mathrm{COCH}_{2}\right)$, 1.89-1.99 (2H, m, $\left.\mathrm{CH}_{2} \mathrm{CH}_{2} \mathrm{CH}_{2}\right), 1.42(9 \mathrm{H}, \mathrm{s}, t-\mathrm{Bu}) ; \delta_{\mathrm{C}}\left(75.5 \mathrm{MHz}, \mathrm{CDCl}_{3}\right) 155.8\left(\mathrm{C}_{\mathrm{q}}\right), 136.6\left(\mathrm{C}_{\mathrm{q}}\right)$, $132.9(\mathrm{CH}), 128.3(\mathrm{CH}), 127.8(\mathrm{CH}), 78.9\left(\mathrm{C}_{\mathrm{q}}\right), 39.9\left(\mathrm{CH}_{2}\right), 35.5\left(\mathrm{CH}_{2}\right), 28.1\left(\mathrm{CH}_{3}\right), 24.3\left(\mathrm{CH}_{2}\right)$; MS $m / z$ (EI) $145\left(\left[\mathrm{M}-\left(t-\mathrm{BuCO}_{2}+\mathrm{H}_{2} \mathrm{O}\right)\right]^{+}\right), 117$ (60), 79 (100), 63 (50); Anal calc: $\mathrm{C}_{15} \mathrm{H}_{21} \mathrm{NO}_{3}$ : : C, 68.42; H, 8.04; N, 5.32. Found: C, 68.35; H, 8.05; N, 5.33.

(5-oxo-5-phenyl-hexyl)-carbamic acid tert-butyl ester (13)<smiles>CC(C)(C)OC(=O)NCCCCCC(=O)c1ccccc1</smiles>

Colourless solid, (0.62 g, $45 \%)$; mp 35-36 ${ }^{\circ} \mathrm{C} ; v_{\max }($ neat $) / \mathrm{cm}^{-1} 3378,2970,2934,2863,2359,2340$, $1701,1674,1512,1447,1364,1250,1157,967,747,725,687,655 ; \delta_{\mathrm{H}}\left(300.13 \mathrm{MHz}, \mathrm{CDCl}_{3}\right) 7.95$ $(2 \mathrm{H}, \mathrm{m}, \operatorname{aryl} \mathrm{H}), 7.55(1 \mathrm{H}, \mathrm{m}$, aryl $\mathrm{H}), 7.46(2 \mathrm{H}, \mathrm{m}, \operatorname{aryl} \mathrm{H}), 4.8(1 \mathrm{H}, \mathrm{s}, \mathrm{NH}), 3.13(2 \mathrm{H}, \mathrm{q}, J 6.4$, $\left.\mathrm{NHCH}_{2}\right), 2.97\left(2 \mathrm{H}, \mathrm{t}, J\right.$ 7.4, $\left.\mathrm{COCH}_{2}\right), 1.76\left(2 \mathrm{H}\right.$, quin, $J$ 7.6, $\left.\mathrm{COCH}_{2} \mathrm{CH}_{2}\right), 1.53\left(2 \mathrm{H}, \mathrm{m}, \mathrm{NHCH}_{2} \mathrm{CH}_{2}\right)$, $1.44(9 \mathrm{H}, \mathrm{s}, t-\mathrm{Bu}), 1.38\left(2 \mathrm{H}, \mathrm{m}, \mathrm{COCH}_{2} \mathrm{CH}_{2} \mathrm{CH}_{2}\right) ; \delta_{\mathrm{C}}\left(75.5 \mathrm{MHz}, \mathrm{CDCl}_{3}\right) 200.1(\mathrm{C}), 156.4(\mathrm{C}), 137.3$ (C), $133.4(\mathrm{CH}), 129.0(\mathrm{CH}), 128.4(\mathrm{CH}), 79.4(\mathrm{C}), 40.8\left(\mathrm{CH}_{2}\right), 38.8\left(\mathrm{CH}_{2}\right), 30.4\left(\mathrm{CH}_{2}\right), 28.8\left(\mathrm{CH}_{3}\right)$, $26.9\left(\mathrm{CH}_{2}\right), 24.3\left(\mathrm{CH}_{2}\right)$; MS m/z (CI) 309 ([M+NH$\left.]^{+}, 24\right), 292$ (27), 253 (73), 235 (100), 192 (80), 174 (58); HRMS: $\mathrm{C}_{17} \mathrm{H}_{26} \mathrm{NO}_{3}(\mathrm{M}+\mathrm{H})$ calc 292.1907, found 292.1906.

\section{(5-oxo-5-(2-thiophene)-pentyl)-carbamic acid tert-butyl ester}<smiles>CC(C)(C)OC(=O)NCCCCC(=O)c1cccs1</smiles>

Pale brown solid, (0.85 g, $72 \%)$; mp 36-38 ${ }^{\circ} \mathrm{C} ; v_{\max }\left(\right.$ neat) $/ \mathrm{cm}^{-1} 3378,2978,2925,2864,1686,1657$, 1242,$1158 ; \delta_{\mathrm{H}}\left(300.13 \mathrm{MHz}, \mathrm{CDCl}_{3}\right)$ 7.70-7.73 $(1 \mathrm{H}, \mathrm{m}$, thiophene $\mathrm{CH}), 7.60-7.63(1 \mathrm{H}, \mathrm{m}, \mathrm{COCH})$, 
7.09-7.14 $(1 \mathrm{H}, \mathrm{m}$, thiophene $\mathrm{CH}), 4.62(1 \mathrm{H}, \mathrm{bs}, \mathrm{NH}), 3.12-3.20\left(2 \mathrm{H}, \mathrm{m}, \mathrm{NHCH}_{2}\right), 2.94(2 \mathrm{H}, \mathrm{t}, J$ 7.2, $\left.\mathrm{COCH}_{2}\right), 1.77-1.78\left(2 \mathrm{H}\right.$, quin, $J$ 7.2, $\left.\mathrm{COCH}_{2} \mathrm{CH}_{2}\right), 1.53-1.62\left(2 \mathrm{H}\right.$, quin, $J$ 7.2, $\left.\mathrm{NHCH}_{2} \mathrm{CH}_{2}\right), 1.43(9 \mathrm{H}$, $\mathrm{s}, t-\mathrm{Bu}) ; \delta_{\mathrm{C}}\left(75.5 \mathrm{MHz}, \mathrm{CDCl}_{3}\right) 156.4\left(\mathrm{C}_{\mathrm{q}}\right), 144.6\left(\mathrm{C}_{\mathrm{q}}\right), 133.9(\mathrm{CH}), 132.3(\mathrm{CH}), 128.5(\mathrm{CH}), 70.4\left(\mathrm{C}_{\mathrm{q}}\right)$, $40.4\left(\mathrm{CH}_{2}\right), 39.1\left(\mathrm{CH}_{2}\right) 29.9\left(\mathrm{CH}_{2}\right), 28.8\left(\mathrm{CH}_{3}\right), 21.9\left(\mathrm{CH}_{2}\right) ; \mathrm{MS} \mathrm{m} / z(\mathrm{CI}) 284\left([\mathrm{M}+\mathrm{H}]^{+}, 15\right), 228(20)$, 166 (100), 165 (80), 111 (50); HRMS: $\mathrm{C}_{14} \mathrm{H}_{22} \mathrm{NO}_{3} \mathrm{~S}$ calc. 284.1320, found 284.1340.

(5-oxo-5-(3-trifluoromethylphenyl)-pentyl)-carbamic acid tert-butyl ester

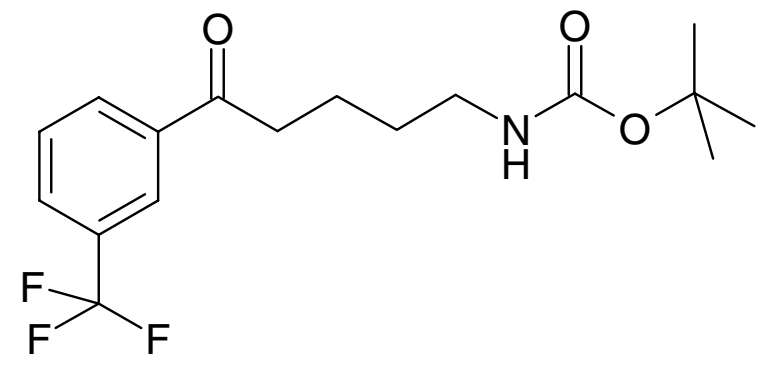

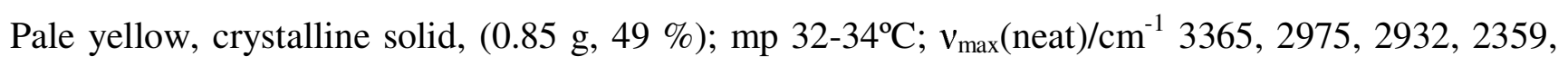
2340, 1686, 1514, 1228, 1163, 1124; $\delta_{\mathrm{H}}\left(300.13 \mathrm{MHz}, \mathrm{CDCl}_{3}\right) 8.21(1 \mathrm{H}, \mathrm{s}$, aryl H), $8.13(1 \mathrm{H} . \mathrm{d}, J$ J.5, $\operatorname{aryl~H}), 7.82(1 \mathrm{H}, \mathrm{d}, J$ 7.5, aryl H), $7.61(1 \mathrm{H}, \mathrm{t}, J$ 7.5, aryl H), $4.62(1 \mathrm{H}, \mathrm{bs}, \mathrm{NH}), 3.12-3.21(2 \mathrm{H}, \mathrm{m}$, $\left.\mathrm{NHCH}_{2}\right), 3.02$, $\left(2 \mathrm{H}, \mathrm{t}, J 7.15, \mathrm{COCH}_{2}\right), 1.77-1.85\left(2 \mathrm{H}\right.$, quin, $\left.J 7.15, \mathrm{COCH}_{2} \mathrm{CH}_{2}\right), 1.55-1.66(2 \mathrm{H}, \mathrm{m}$, $\left.\mathrm{NHCH}_{2} \mathrm{CH}_{2}\right), 1.48(9 \mathrm{H}, \mathrm{s}, t-\mathrm{Bu}) ; \delta_{\mathrm{C}}\left(75.5 \mathrm{MHz}, \mathrm{CDCl}_{3}\right) 156.6\left(\mathrm{C}_{\mathrm{q}}\right), 137.7\left(\mathrm{C}_{\mathrm{q}}\right), 131.6(\mathrm{CH}), 129.8$ $(\mathrm{CH}), 129.7(\mathrm{CH}), 125.2(\mathrm{CH}), 79.6\left(\mathrm{C}_{\mathrm{q}}\right), 40.5\left(\mathrm{CH}_{2}\right), 38.5\left(\mathrm{CH}_{2}\right), 29.9\left(\mathrm{CH}_{2}\right), 28.8\left(\mathrm{CH}_{3}\right), 21.3$ $\left(\mathrm{CH}_{2}\right) ; \mathrm{MS} m / z$ (EI) $346\left([\mathrm{M}+\mathrm{H}]^{+}, 35\right), 289$ (45), 271 (65), 227 (55), 82 (100); Anal. calc. for $\mathrm{C}_{17^{-}}$ $\mathrm{H}_{22} \mathrm{~F}_{3} \mathrm{NO}_{3}$ : C, 59.12; H, 6.42; N, 4.04, found C, 58.92; H, 6.47; N, 4.01 .

(5-oxo-5-(4-trifluoromethylphenyl)-pentyl)-carbamic acid tert-butyl ester

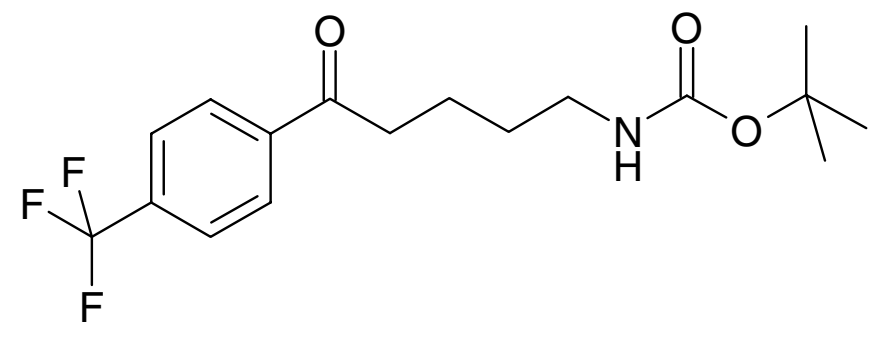

Pale yellow solid, (0.48 g, $28 \%$ ); mp 79-81 ${ }^{\circ} \mathrm{C} ; v_{\max }$ (neat) $/ \mathrm{cm}^{-1} 3368,2980,2939,2863,2357,1673$, $1509,1321,1161,1062 ; \delta_{\mathrm{H}}\left(300.13 \mathrm{MHz}, \mathrm{CDCl}_{3}\right) 8.03(2 \mathrm{H}, \mathrm{d}, J$ 8.4, aryl H), $7.71(2 \mathrm{H}, \mathrm{d}, J$ 8.4, aryl $\mathrm{H}), 4.70(1 \mathrm{H}, \mathrm{bs}, \mathrm{NH}), 3.12-3.22\left(2 \mathrm{H}, \mathrm{m}, \mathrm{NHCH}_{2}\right), 3.02\left(2 \mathrm{H}, \mathrm{t}, J \mathrm{~J}, 3, \mathrm{COCH}_{2}\right), 1.78-1.86(2 \mathrm{H}, \mathrm{m}$, $\left.\mathrm{COCH}_{2} \mathrm{CH}_{2}\right), 1.58-1.67\left(2 \mathrm{H}, \mathrm{m}, \mathrm{NHCH}_{2} \mathrm{CH}_{2}\right), 1.42(9 \mathrm{H}, \mathrm{s}, t-\mathrm{Bu}) ; \delta_{\mathrm{C}}\left(75.5 \mathrm{MHz}, \mathrm{CDCl}_{3}\right) 156.6\left(\mathrm{C}_{\mathrm{q}}\right)$, $140.0\left(\mathrm{C}_{\mathrm{q}}\right), 128.7(\mathrm{CH}), 126.1(\mathrm{CH}), 79.8\left(\mathrm{C}_{\mathrm{q}}\right), 40.5,\left(\mathrm{CH}_{2}\right), 38.6\left(\mathrm{CH}_{2}\right), 29.9\left(\mathrm{CH}_{2}\right), 28.9\left(\mathrm{CH}_{3}\right), 21.4$ 
$\left(\mathrm{CH}_{2}\right) ; \mathrm{MS} \mathrm{m} / z$ (EI) $346\left([\mathrm{M}+\mathrm{H}]^{+}, 45\right), 290$ (60), 272 (45), 246 (100), 228 (60), 173 (65), 145 (50);

HRMS: $\mathrm{C}_{17} \mathrm{H}_{23} \mathrm{~F}_{3} \mathrm{NO}_{3}$ calc. 346.1630, found 346.1629; Anal. Calc. for $\mathrm{C}_{17} \mathrm{H}_{22} \mathrm{~F}_{3} \mathrm{NO}_{3}$ : C, 59.12; $\mathrm{H}$, 6.42; N, 4.04, found C, 59.03; H, 6.46; N, 3.97 .

\section{General procedure for the racemic preparation of amines}<smiles>[R]C(=O)C(C)(C)CCCNC(=O)OC(C)(C)C</smiles>

The $t$-Boc-amino-ketone ( $4 \mathrm{mmol}$ ) was stirred in TFA for $2 \mathrm{hrs}$. TLC was used to check the reaction progress. $50 \% \mathrm{w} / \mathrm{v} \mathrm{NaOH}$ solution was added to the mixture until the $\mathrm{pH}$ was $13-14$. The product was extracted 4 x 20mL with DCM and the organic layers combined, dried with $\mathrm{MgSO}_{4}$ and evaporated. The product was dissolved in water / methanol $(1: 4,5 \mathrm{~mL})$ and added to a flask followed by $\mathrm{NaBH}_{4}$ ( $4.5 \mathrm{mmol}$ ). The mixture was stirred under $\mathrm{N}_{2}$ overnight. The mixture was acidified with $1-2 \mathrm{M} \mathrm{HCl}$ until the $\mathrm{pH}$ was 1-3 and left for 30 minutes. $\mathrm{NaOH}$ solution was then added until the $\mathrm{pH}$ was 13-14 and the product was extracted with DCM (4 x $20 \mathrm{~mL})$, the organic layers were combined, dried with $\mathrm{MgSO}_{4}$, filtered and evaporated to yield the product.

\section{(士)-2-phenyl-piperidine $(7)^{2}$}<smiles>c1ccc([C@H]2CCCCN2)cc1</smiles>

Yellow liquid, (70 mg, 60\%); $v_{\max }\left(\right.$ neat) $/ \mathrm{cm}^{-1} 3321,3268,3060,3025,2929,2850,2784,2697,1945$, 1599, 1490, 1440, 1324, 1307, 1107, 1019, 749, 697; $\delta_{\mathrm{H}}\left(300.13 \mathrm{MHz}, \mathrm{CDCl}_{3}\right) 7.29(5 \mathrm{H}, \mathrm{m}$, aryl H), $3.58(1 \mathrm{H}, \mathrm{m}, \mathrm{NHCH}), 3.19\left(1 \mathrm{H}, \mathrm{m}, \mathrm{NHCH}_{\mathrm{a}} \mathrm{H}_{\mathrm{b}}\right), 2.80\left(1 \mathrm{H}, \mathrm{m}, \mathrm{NHCH}_{\mathrm{a}} H_{\mathrm{b}}\right), 1.85\left(2 \mathrm{H}, \mathrm{m}, \mathrm{CH}_{2}\right), 1.66$ $\left(2 \mathrm{H}, \mathrm{m}, \mathrm{CH}_{2}\right), 1.52\left(3 \mathrm{H}, \mathrm{m}, \mathrm{NH}\right.$ and $\left.\mathrm{CH}_{2}\right) ; \delta_{\mathrm{C}}\left(75.5 \mathrm{MHz}, \mathrm{CDCl}_{3}\right) 145.9(\mathrm{C}), 128.8(\mathrm{CH}), 127.4(\mathrm{CH})$, $127.0(\mathrm{CH}), 62.8(\mathrm{CH}), 48.2\left(\mathrm{CH}_{2}\right), 35.4\left(\mathrm{CH}_{2}\right), 26.3\left(\mathrm{CH}_{2}\right), 25.8\left(\mathrm{CH}_{2}\right) ; \mathrm{MS} \mathrm{m} / z(\mathrm{EI}) 161\left([\mathrm{M}]^{+}, 57\right)$, 160 (47), 132 (76), 118 (43), 104 (100), 91 (48), 84 (44), 77 (28). HRMS: $\mathrm{C}_{11} \mathrm{H}_{15} \mathrm{~N}$ calc. 161.1205, found 161.1197; Anal. calc. for $\mathrm{C}_{11} \mathrm{H}_{15} \mathrm{~N}$ : C, 81.94; H, 9.38; N, 8.69, found C, 81.70; H, 9.38; N, 8.77.

\section{(士)-2-cyclohexyl-piperidine}


<smiles>C1CCC([C@@H]2CCCCN2)CC1</smiles>

Yellow liquid, (50 mg, $68 \%$ ); $v_{\max }\left(\right.$ neat) $/ \mathrm{cm}^{-1}$ 3344, 2920, 2851, 2698, 2502, 2408, 2358, 1673, 1447 , 1200, 1175, 1130, 798, 719; $\delta_{\mathrm{H}}\left(300 \mathrm{MHz} \mathrm{CDCl}_{3}\right) 3.12(1 \mathrm{H}$, dquin, J 11.7, 2.1, NHCH), $2.9(1 \mathrm{H}, \mathrm{s}$, $\mathrm{NH}), 2.62\left(1 \mathrm{H}, \mathrm{td}, J 11.7,2.9, \mathrm{NHCH}_{\mathrm{a}} \mathrm{H}_{\mathrm{b}}\right), 2.26\left(1 \mathrm{H}, \mathrm{m}, \mathrm{NHCH}_{\mathrm{a}} H_{\mathrm{b}}\right), 1.71(8 \mathrm{H}, \mathrm{m}, 4 \mathrm{xCH}), 1.21(7 \mathrm{H}$, m, $\mathrm{NH}$ and $\left.3 \mathrm{xCH}_{2}\right), 1.00\left(2 \mathrm{H}, \mathrm{m}, \mathrm{CH}_{2}\right) ; \delta_{\mathrm{C}}\left(75.5 \mathrm{MHz}, \mathrm{CDCl}_{3}\right) 62.5(\mathrm{CH}), 47.8\left(\mathrm{CH}_{2}\right), 43.6(\mathrm{CH})$, $29.7\left(\mathrm{CH}_{2}\right), 29.5\left(\mathrm{CH}_{2}\right), 27.0\left(\mathrm{CH}_{2}\right), 26.8\left(\mathrm{CH}_{2}\right), 26.7\left(\mathrm{CH}_{2}\right), 25.3\left(\mathrm{CH}_{2}\right) ; \mathrm{MS} m / z(\mathrm{EI}) 166$ $\left([\mathrm{M}-\mathrm{H}]^{+}, 1\right), 149$ (2), 84 (100), 78 (15), 63 (11); HRMS: $\mathrm{C}_{11} \mathrm{H}_{22} \mathrm{~N}$ calc. 168.1752, found 168.1758.

\section{(士)-2-(2-methoxyphenyl)-piperidine}<smiles>COc1ccccc1[C@H]1CCCCN1</smiles>

Pale yellow oil, (66 mg, $84 \%) ; v_{\max }\left(\right.$ neat) $/ \mathrm{cm}^{-1} 2928,2850,2833,2782,2720,1600,1586,1490$, $1461,1437,1236,1107,1049,1022,750,627 ; \delta_{\mathrm{H}}\left(300.13 \mathrm{MHz}, \mathrm{CDCl}_{3}\right) 7.43(1 \mathrm{H}, \mathrm{dd}, J$ 7.7, 1.7, aryl H), $7.20(1 \mathrm{H}, \mathrm{td}, J$ 7.7, 1.7, aryl H), $6.94(1 \mathrm{H}, \mathrm{t}, J$ 7.5, aryl H), $6.84(1 \mathrm{H}, \mathrm{d}, J$ 8.3, aryl H), $3.96(1 \mathrm{H}$, $\mathrm{dd}, J$ 2.4, 10.4, $\mathrm{NHCH}), 3.82\left(3 \mathrm{H}, \mathrm{s}, \mathrm{OCH}_{3}\right), 3.17\left(1 \mathrm{H}, \mathrm{d}, J 11.7, \mathrm{NHCH}_{\mathrm{a}} \mathrm{CH}_{\mathrm{b}}\right), 2.81(1 \mathrm{H}, \mathrm{m}$, $\left.\mathrm{NHCH}_{\mathrm{a}} \mathrm{CH}_{\mathrm{b}}\right), 2.35(1 \mathrm{H}, \mathrm{s}, \mathrm{NH}), 1.85\left(2 \mathrm{H}, \mathrm{m}, \mathrm{CH}_{2}\right), 1.53\left(4 \mathrm{H}, \mathrm{m}, 2 \mathrm{xCH}_{2}\right) ; \delta_{\mathrm{C}}\left(75.5 \mathrm{MHz}, \mathrm{CDCl}_{3}\right)$ $156.9(\mathrm{C}), 133.4(\mathrm{C}), 128.1(\mathrm{CH}), 127.2(\mathrm{CH}), 121.1(\mathrm{CH}), 110.6(\mathrm{CH}), 55.7\left(\mathrm{CH}_{3}\right), 48.2\left(\mathrm{CH}_{2}\right), 33.1$ $\left(\mathrm{CH}_{2}\right), 26.5\left(\mathrm{CH}_{2}\right), 25.8\left(\mathrm{CH}_{2}\right) ; \mathrm{MS} \mathrm{m} / z(\mathrm{EI}) 191$ ([M] $\left.]^{+}, 100\right), 162$ (63), 134 (25), 119 (18), 91 (12), 77 (4); HRMS: $\mathrm{C}_{12} \mathrm{H}_{18} \mathrm{NO}(\mathrm{M}+\mathrm{H})$, calc.192.1383, found 192.1383.

\section{(士)-2-(3-methoxyphenyl)-piperidine}




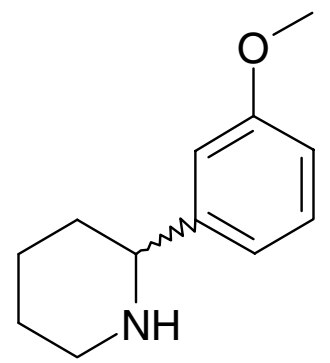

Pale yellow oil, (38 mg, $51 \%$ ); $v_{\max }\left(\right.$ neat) $/ \mathrm{cm}^{-1} 3313,2929,2850,2783,2718,2359,2340,1600$, $1583,1485,1431,1261,1157,1042,845,778,697 ; \delta_{\mathrm{H}}\left(300.13 \mathrm{MHz}, \mathrm{CDCl}_{3}\right) 7.20(1 \mathrm{H}, \mathrm{m}$, aryl H), $6.93(2 \mathrm{H}, \mathrm{m}, \operatorname{aryl} \mathrm{H}), 6.78\left(1 \mathrm{H}, \mathrm{m}\right.$, aryl H), $3.80\left(3 \mathrm{H}, \mathrm{s}, \mathrm{OCH}_{3}\right), 3.56(1 \mathrm{H}, \mathrm{m}, \mathrm{NHCH}), 3.17(1 \mathrm{H}, \mathrm{m}$, $\left.\mathrm{NHCH}_{\mathrm{a}} \mathrm{CH}_{\mathrm{b}}\right), 2.77\left(1 \mathrm{H}, \mathrm{td}, J\right.$ 11.5, 3.0, $\left.\mathrm{NHCH}_{\mathrm{a}} \mathrm{CH}_{\mathrm{b}}\right), 2.14(1 \mathrm{H}, \mathrm{s}, \mathrm{NH}), 1.87(1 \mathrm{H}, \mathrm{m}, \mathrm{CH}), 1.78(1 \mathrm{H}$, m, $\mathrm{CH}), 1.63(1 \mathrm{H}, \mathrm{m}, \mathrm{CH}), 1.50(3 \mathrm{H}, \mathrm{m}, 3 \mathrm{x} \mathrm{CH}) ; \delta_{\mathrm{C}}\left(75.5 \mathrm{MHz}, \mathrm{CDCl}_{3}\right) 160.1(\mathrm{C}), 147.6(\mathrm{C}), 129.7$ $(\mathrm{CH}), 119.4(\mathrm{CH}), 113.1(\mathrm{CH}), 112.3(\mathrm{CH}), 62.8(\mathrm{CH}), 55.6\left(\mathrm{CH}_{3}\right), 48.2\left(\mathrm{CH}_{2}\right), 35.3\left(\mathrm{CH}_{2}\right), 26.2$ $\left(\mathrm{CH}_{2}\right), 25.8\left(\mathrm{CH}_{2}\right) ; \mathrm{MS} \mathrm{m} / z(\mathrm{EI}) 191\left([\mathrm{M}]^{+}, 100\right), 190$ (54), 162 (71), 148 (40), 134 (84), 84 (55); HRMS: $\mathrm{C}_{12} \mathrm{H}_{17} \mathrm{NO}$, calc. 191.1310, found 191.1324.

\section{(士)-2-(4-methoxyphenyl)-piperidine ${ }^{4}$}<smiles>COc1ccc([C@@H]2CCCCN2)cc1</smiles>

Pale yellow oil, (58 mg, 74\%); $v_{\max }$ (neat)/cm $\mathrm{cm}^{-1}$ 3324, 3268, 2996, 2928, 2849, 2782, 2716, 2359, $1609,1510,1440,1302,1238,1172,1109,1035,826,761,636 ; \delta_{\mathrm{H}}\left(300 \mathrm{MHz}, \mathrm{CDCl}_{3}\right) 7.28(2 \mathrm{H}, \mathrm{d}, J$ 8.9, aryl H), $6.85\left(2 \mathrm{H}, \mathrm{d}, J\right.$ 8.3, aryl H), $3.79\left(3 \mathrm{H}, \mathrm{s}, \mathrm{OCH}_{3}\right), 3.53(1 \mathrm{H}, \mathrm{m}, \mathrm{NHCH}), 3.17(1 \mathrm{H}, \mathrm{d}, J 11.3$, $\left.\mathrm{NHCH}_{\mathrm{a}} \mathrm{H}_{\mathrm{b}}\right), 2.78\left(1 \mathrm{H}, \mathrm{td}, J 11.3,2.3, \mathrm{NHCH}_{\mathrm{a}} H_{\mathrm{b}}\right), 1.97(1 \mathrm{H}, \mathrm{s}, \mathrm{NH}), 1.89(1 \mathrm{H}, \mathrm{m}, \mathrm{CH}), 1.74(1 \mathrm{H}, \mathrm{m}$, $\mathrm{CH}), 1.63(1 \mathrm{H}, \mathrm{m}, \mathrm{CH}), 1.49(3 \mathrm{H}, \mathrm{m}, \mathrm{CH}) ; \delta_{\mathrm{C}}\left(75.5 \mathrm{MHz}, \mathrm{CDCl}_{3}\right) 158.4(\mathrm{C}), 137.5(\mathrm{C}), 127.5(\mathrm{CH})$, $113.4(\mathrm{CH}), 61.5(\mathrm{CH}), 55.0\left(\mathrm{CH}_{3}\right), 47.6\left(\mathrm{CH}_{2}\right), 34.7\left(\mathrm{CH}_{2}\right), 25.6\left(\mathrm{CH}_{2}\right), 25.2\left(\mathrm{CH}_{2}\right) ; \mathrm{MS} m / z(\mathrm{EI}) 191$ ([M] $\left.]^{+}, 100\right), 162$ (76), 135 (80), 121 (27), 91 (22), 77 (16); HRMS: calc. $\mathrm{C}_{12} \mathrm{H}_{16} \mathrm{NO}$ 190.1231, found 190.1251.

\section{(土)-2-phenyl-cycloheptylamine ${ }^{2}$}




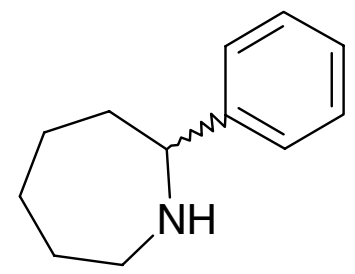

Colourless oil, (75 mg, $83 \%$ ); $v_{\max }\left(\right.$ neat) $/ \mathrm{cm}^{-1}$ 3059, 3025, 2921, 2850, 2358, 2335, 1491, 1449, 1395, 1332, 1268, 1211, 1142, 753, 697; $\delta_{\mathrm{H}}\left(300.13 \mathrm{MHz}, \mathrm{CDCl}_{3}\right) 7.25(5 \mathrm{H}, \mathrm{m}$, aryl H), $3.75(1 \mathrm{H}, \mathrm{dd}, J 2.2$, 10.1, NHCH), $3.12\left(1 \mathrm{H}, \mathrm{dt}, J 15.3,3.6, \mathrm{NHCH}_{\mathrm{a}} \mathrm{H}_{\mathrm{b}}\right), 2.86\left(1 \mathrm{H}, \mathrm{m}, \mathrm{NHCH}_{\mathrm{a}} H_{\mathrm{b}}\right), 1.95(1 \mathrm{H}, \mathrm{m}, \mathrm{CH}), 1.71$ $(7 \mathrm{H}, \mathrm{m}, 7 \mathrm{xCH}) ; \delta_{\mathrm{C}}\left(75.5 \mathrm{MHz}, \mathrm{CDCl}_{3}\right) 148.2(\mathrm{C}), 128.8(\mathrm{CH}), 126.8(\mathrm{CH}), 126.3(\mathrm{CH}), 65.3(\mathrm{CH})$, $48.6\left(\mathrm{CH}_{2}\right), 39.4\left(\mathrm{CH}_{2}\right), 31.2\left(\mathrm{CH}_{2}\right), 27.3\left(\mathrm{CH}_{2}\right), 26.1\left(\mathrm{CH}_{2}\right) ; \mathrm{MS} m / z(\mathrm{EI}) 175\left([\mathrm{M}]^{+}, 64\right), 132(100)$, 118 (70), 104 (27), 91 (33); HRMS: $\mathrm{C}_{12} \mathrm{H}_{17} \mathrm{~N}$ calc. 175.1361, found 175.1359.

\section{(士)-2-Thiophenyl-2-yl-piperidine}

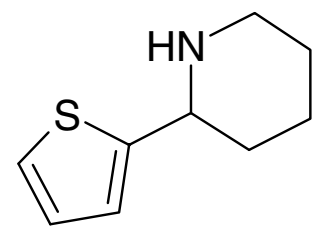

Colourless oil, (52 mg, $79 \%) ; v_{\max }\left(\right.$ neat) $/ \mathrm{cm}^{-1}$ 3310, 2930, 2850, 2789, 2359, 2341, 1439, 1308, 1105; $\delta_{\mathrm{H}}$ 7.16-7.12 $(1 \mathrm{H}, \mathrm{m}$, thiophene $\mathrm{CH}), 6.89-6.93(2 \mathrm{H}, \mathrm{m}$, thiophene $\mathrm{CH}), 3.85-3.91(1 \mathrm{H}, \mathrm{m}, \mathrm{NHCH})$, 3.10-3.17 $\left(1 \mathrm{H}, \mathrm{m}, \mathrm{NHCH}_{\mathrm{a}} \mathrm{CH}_{\mathrm{b}}\right), 2.71-2.79\left(1 \mathrm{H}, \mathrm{m}, \mathrm{NHCH}_{\mathrm{a}} \mathrm{CH}_{\mathrm{b}}\right), 1.82-2.96\left(3 \mathrm{H}, \mathrm{m}, \mathrm{NH}+\mathrm{CH}_{2}\right) 1.42-$ $1.63\left(4 \mathrm{H}, \mathrm{m}, 2 \times \mathrm{CH}_{2}\right) ; \delta_{\mathrm{C}}\left(75.5 \mathrm{MHz}, \mathrm{CDCl}_{3}\right) 150.1\left(\mathrm{C}_{\mathrm{q}}\right), 126.7(\mathrm{CH}), 123.9(\mathrm{CH}), 122.9(\mathrm{CH}), 57.7$ $(\mathrm{CH}), 47.9\left(\mathrm{CH}_{2}\right), 36.3\left(\mathrm{CH}_{2}\right), 26.3\left(\mathrm{CH}_{2}\right), 25.4\left(\mathrm{CH}_{2}\right)$; MS m/z (EI) $167\left(\mathrm{M}^{+}, 10\right), 85$ (70), 83 (100); HRMS: calc. $\mathrm{C}_{9} \mathrm{H}_{13} \mathrm{NS}$ calc. 167.0769, found 167.0769.

\section{(土)-2-(3-Trifluoromethylphenyl)-piperidine}

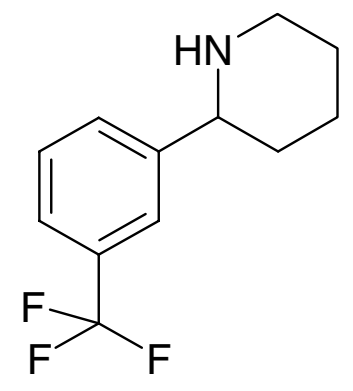

Pale yellow oil (59 mg, 65\%); $v_{\max }$ (neat) $/ \mathrm{cm}^{-1} 3271,2932,2853,1320,1117,702 ; \delta_{\mathrm{H}} 7.63$ (s, 1H, aryl H), 7.39-7.59 (3H, m, aryl H), 3.60-3.68 $(1 \mathrm{H}, \mathrm{m}, \mathrm{NHCH}), 3.17-3.26\left(1 \mathrm{H}, \mathrm{m}, \mathrm{NHCH}_{\mathrm{a}} \mathrm{CH}_{\mathrm{b}}\right), 2.76-2.84$ 
$\left(1 \mathrm{H}, \mathrm{m}, \mathrm{NHCH}_{\mathrm{a}} \mathrm{CH} \mathrm{b}_{\mathrm{b}}\right), 1.47-1.92\left(7 \mathrm{H}, \mathrm{m}, \mathrm{NHCH}_{2} \mathrm{CH}_{2} \mathrm{CH}_{2} \mathrm{CH}_{2}\right) ; \delta_{\mathrm{C}}\left(75.5 \mathrm{MHz}, \mathrm{CDCl}_{3}\right) 146.8\left(\mathrm{C}_{\mathrm{q}}\right)$, $130.6\left(\mathrm{C}_{\mathrm{q}}, \mathrm{q}, J 24\right), 130.0(\mathrm{CH}), 129.1(\mathrm{CH}), 124.6(\mathrm{CH}, \mathrm{d}, J 272), 124.3(\mathrm{CH}), 123.9(\mathrm{CH}), 62.3(\mathrm{CH})$, $\left.48.0\left(\mathrm{CH}_{2}\right), 35.4\left(\mathrm{CH}_{2}\right), 26.1\left(\mathrm{CH}_{2}\right), 25.6\left(\mathrm{CH}_{2}\right) ;\right) ; \mathrm{MS} \mathrm{m/z} \mathrm{(EI)} 229\left(\mathrm{M}^{+}, 65\right), 228(55), 200(80), 172$ (100), 84 (86); HRMS: calc. $\mathrm{C}_{12} \mathrm{H}_{13} \mathrm{~F}_{3} \mathrm{~N}$ calc. 228.1000, found 228.0988.

\section{( \pm )-2-(4-Trifluoromethylphenyl)-piperidine}

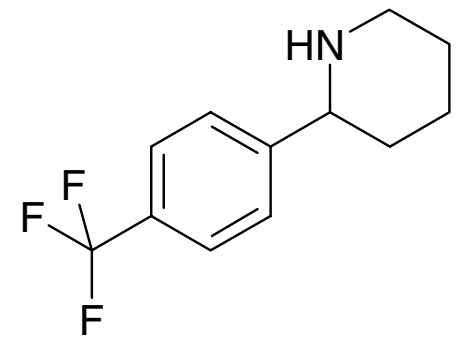

Pale yellow oil (52 mg, 57\%); $v_{\max }$ (neat)/ $/ \mathrm{cm}^{-1} 3675,2933,2854,2790,1619,1321,1117,1066 ; \delta_{\mathrm{H}}$ $7.55(2 \mathrm{H}, \mathrm{d}, J$ 8.1, aryl H), $7.48(2 \mathrm{H}, \mathrm{d}, J$ 8.1, aryl H), 3.61-3.68 $(1 \mathrm{H}, \mathrm{m}, \mathrm{NHCH}), 3.27-3.32(1 \mathrm{H}, \mathrm{m}$, $\left.\mathrm{NHCH}_{\mathrm{a}} \mathrm{CH}_{\mathrm{b}}\right), 2.76-2.84\left(1 \mathrm{H}, \mathrm{m}, \mathrm{NHCH}_{\mathrm{a}} \mathrm{CH}_{\mathrm{b}}\right), 1.95-1.42\left(7 \mathrm{H}, \mathrm{m}, \mathrm{NHCH} \mathrm{CH}_{2} \mathrm{CH}_{2}\right) ; \delta_{\mathrm{C}}(75.5 \mathrm{MHz}$, $\left.\mathrm{CDCl}_{3}\right) 149.9\left(\mathrm{C}_{\mathrm{q}}\right), 129.6\left(\mathrm{C}_{\mathrm{q}}, \mathrm{q}, J 32\right), 127.3(\mathrm{CH}), 125.7(\mathrm{CH}), 124.6\left(\mathrm{C}_{\mathrm{q}}, \mathrm{q}, J 272\right), 62.3\left(\mathrm{C}_{\mathrm{q}}\right), 47.9$ $\left(\mathrm{CH}_{2}\right), 35.44\left(\mathrm{CH}_{2}\right), 26.1\left(\mathrm{CH}_{2}\right), 25.6\left(\mathrm{CH}_{2}\right) ; \mathrm{m} / z(\mathrm{EI}) 229\left(\mathrm{M}^{+}, 40\right), 200$ (75), 186 (35), 162 (100), 84 (95), 78 (40), 63 (50); HRMS: calc. $\mathrm{C}_{12} \mathrm{H}_{13} \mathrm{~F}_{3} \mathrm{~N}$ calc. 228.1000, found 228.0993.

\section{Preparation (One-pot process) of piperidines.}
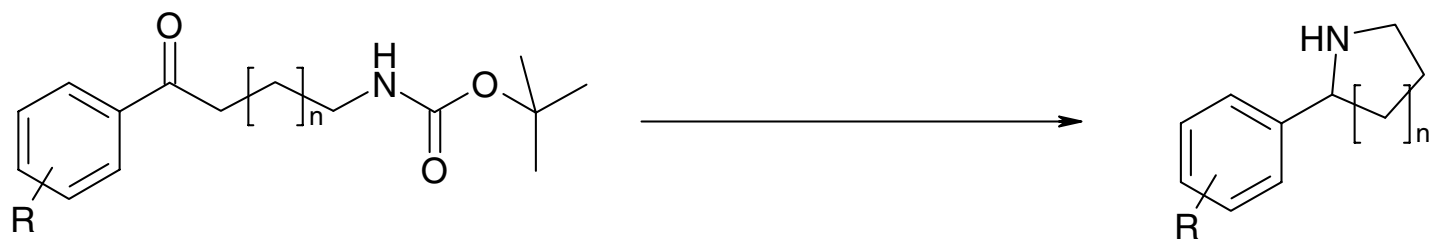

$t$-Boc-amino-ketone $(0.2 \mathrm{~g})$ was stirred in freshly distilled formic acid $(1.8 \mathrm{~mL})$ for $16 \mathrm{~h}$. The flask was then sealed and cooled to $0^{\circ} \mathrm{C}$; triethylamine $(3 \mathrm{~mL})$ was added cautiously with vigorous shaking until all gas had redissolved. In a separate flask a mixture of (p-cymene) ruthenium (II) chloride dimmer $(0.25 \mathrm{~mol} \%)$ and $(1 R, 2 R)$-TsDPEN $(0.5 \mathrm{~mol} \%)$, triethylamine (1 drop) and anhydrous acetonitrile (1 $\mathrm{mL}$ ) were stirred at $28^{\circ} \mathrm{C}$ for $40 \mathrm{~min}$. The catalyst solution was transferred to the formic acid / triethylamine solution and the mixture stirred at $28^{\circ} \mathrm{C}$ until complete by NMR. The mixture was made basic (pH 9-10) with sat. $\mathrm{Na}_{2} \mathrm{CO}_{3}$ sol. and extracted with DCM $(3 \times 25 \mathrm{~mL})$. The combined organics were dried $\left(\mathrm{MgSO}_{4}\right)$, filtered and the solvent removed under reduced pressure. The residue was 
purified by flash column chromatography (10-15\% v/v ethyl acetate / hexane on silica pre-treated with $\mathrm{Et}_{3} \mathrm{~N}$ ) to afford the amines as described.

\section{2-phenyl-piperidine (7)}

Colourless oil, (110 mg, 94\%); $\delta_{\mathrm{H}}\left(300.13 \mathrm{MHz}, \mathrm{CDCl}_{3}\right) 7.29(5 \mathrm{H}, \mathrm{m}$, aryl H), $3.58(1 \mathrm{H}, \mathrm{m}, \mathrm{NHCH})$, $3.19\left(1 \mathrm{H}, \mathrm{m}, \mathrm{NHCH}_{\mathrm{a}} \mathrm{H}_{\mathrm{b}}\right), 2.80\left(1 \mathrm{H}, \mathrm{m}, \mathrm{NHCH}_{\mathrm{a}} H_{\mathrm{b}}\right), 1.85\left(2 \mathrm{H}, \mathrm{m}, \mathrm{CH}_{2}\right), 1.66\left(2 \mathrm{H}, \mathrm{m}, \mathrm{CH}_{2}\right), 1.52(3 \mathrm{H}$, $\mathrm{m}, \mathrm{NH}$ and $\mathrm{CH}_{2}$ ).

\section{2-cyclohexyl-piperidine}

Pale orange oil, (115 mg, 98\%); $\delta_{\mathrm{H}}\left(300.13 \mathrm{MHz}, \mathrm{CDCl}_{3}\right) 3.12(1 \mathrm{H}$, dquin, $J$ 11.7, 2.1, NHCH), 2.62 $\left(1 \mathrm{H}, \mathrm{td}, J 11.7,2.9, \mathrm{NHCH}_{\mathrm{a}} \mathrm{H}_{\mathrm{b}}\right), 2.26\left(1 \mathrm{H}, \mathrm{m}, \mathrm{NHCH}_{\mathrm{a}} H_{\mathrm{b}}\right), 1.71(8 \mathrm{H}, \mathrm{m}, 7 \mathrm{xCH}$ and $\mathrm{NH}), 1.21(8 \mathrm{H}, \mathrm{m}$, $8 \mathrm{xCH}), 1.00(2 \mathrm{H}, \mathrm{m}, 2 \mathrm{xCH})$.

\section{2-(2-methoxyphenyl)-piperidine}

Yellow oil, (97 mg, 78\%); $\delta_{\mathrm{H}}\left(300.13 \mathrm{MHz}, \mathrm{CDCl}_{3}\right) 7.43(1 \mathrm{H}, \mathrm{dd}, J$ 7.7, 1.7, aryl H), $7.20(1 \mathrm{H}, \mathrm{td}, J$ 7.7, 1.7, aryl H), $6.94(1 \mathrm{H}, \mathrm{t}, J$ 7.5, aryl H), $6.84(1 \mathrm{H}, \mathrm{d}, J$ 8.3, aryl H), $3.96(1 \mathrm{H}, \mathrm{dd}, J$ 2.4, 10.4, $\mathrm{NHCH}), 3.82\left(3 \mathrm{H}, \mathrm{s}, \mathrm{OCH}_{3}\right), 3.25(1 \mathrm{H}, \mathrm{s}, \mathrm{NH}), 3.17\left(1 \mathrm{H}, \mathrm{d}, J 11.7, \mathrm{NHCH}_{\mathrm{a}} \mathrm{H}_{\mathrm{b}}\right), 2.81(1 \mathrm{H}, \mathrm{td}, J 11.7$, 2.6, $\left.\mathrm{NHCH}_{\mathrm{a}} \mathrm{H}_{\mathrm{b}}\right), 1.85\left(2 \mathrm{H}, \mathrm{m}, \mathrm{CH}_{2}\right), 1.53\left(4 \mathrm{H}, \mathrm{m}, 2 \mathrm{xCH} \mathrm{H}_{2}\right)$.

\section{2-(3-methoxyphenyl)-piperidine}

Pale yellow oil, (120 mg, 96\%); $\delta_{\mathrm{H}}\left(300.13 \mathrm{MHz}, \mathrm{CDCl}_{3}\right) 7.20(1 \mathrm{H}, \mathrm{t}, J$ 7.8, aryl H), 7.03 (1H, s, aryl H), $6.97\left(1 \mathrm{H}, \mathrm{d}, J 7.9\right.$, aryl H), $6.78\left(1 \mathrm{H}, \mathrm{dd}, J 7.8,1.8\right.$, aryl H), $3.75\left(3 \mathrm{H}, \mathrm{s}, \mathrm{OCH}_{3}\right), 3.00(1 \mathrm{H}, \mathrm{m}$, $\mathrm{NHCH}), 2.62\left(1 \mathrm{H}, \mathrm{td}, J\right.$ 11.5, 3.0, $\left.\mathrm{NHCH}_{\mathrm{a}} \mathrm{H}_{\mathrm{b}}\right), 1.90\left(3 \mathrm{H}, \mathrm{m}, \mathrm{NHCH}_{\mathrm{a}} H_{\mathrm{b}}\right.$ and $\left.\mathrm{CH}_{2}\right), 1.68(3 \mathrm{H}, \mathrm{m}, \mathrm{N} H$ and $\left.\mathrm{CH}_{2}\right), 1.52\left(2 \mathrm{H}, \mathrm{m}, \mathrm{CH}_{2}\right)$.

\section{2-(4-methoxyphenyl)-piperidine}

Brown oil, (117 mg, 94\%); $\delta_{\mathrm{H}}\left(300.13 \mathrm{MHz}, \mathrm{CDCl}_{3}\right) 7.28(2 \mathrm{H}, \mathrm{d}, J$ 8.9, aryl H), 6.85 (2H, d, $J$ 8.3, aryl H), $5.05(1 \mathrm{H}, \mathrm{s}, \mathrm{NH}), 3.79\left(3 \mathrm{H}, \mathrm{s}, \mathrm{OCH}_{3}\right), 3.59\left(1 \mathrm{H}, \mathrm{m}, \mathrm{NHCH}_{\mathrm{a}} \mathrm{H}_{\mathrm{b}}\right), 3.09(1 \mathrm{H}, \mathrm{d}, J$ 11.3, $\left.\mathrm{NHCH}_{\mathrm{a}} H_{\mathrm{b}}\right), 2.78(1 \mathrm{H}, \mathrm{m}, \mathrm{CH}), 1.89(1 \mathrm{H}, \mathrm{m}, \mathrm{CH}), 1.78(1 \mathrm{H}, \mathrm{m}, \mathrm{CH}), 1.61\left(3 \mathrm{H}, \mathrm{m}, \mathrm{CH}\right.$ and $\left.\mathrm{CH}_{2}\right), 1.48$ $(1 \mathrm{H}, \mathrm{m}, \mathrm{CH})$.

\section{2-(3-Trifluoromethylphenyl)-piperidine}


Yellow oil, (131 mg, 99\%), $\delta_{\mathrm{H}}\left(300.13 \mathrm{MHz}, \mathrm{CDCl}_{3}\right) 7.63(\mathrm{~s}, 1 \mathrm{H}$, aryl H), 7.39-7.59 (3H, m, aryl H), 3.60-3.68 $(1 \mathrm{H}, \mathrm{m}, \mathrm{NHCH}), 3.17-3.26\left(1 \mathrm{H}, \mathrm{m}, \mathrm{NHCH}_{\mathrm{a}} \mathrm{CH}_{\mathrm{b}}\right), 2.76-2.84\left(1 \mathrm{H}, \mathrm{m}, \mathrm{NHCH}_{\mathrm{a}} \mathrm{CH}_{\mathrm{b}}\right), 1.47-$ $1.92\left(7 \mathrm{H}, \mathrm{m}, \mathrm{NHCH}_{2} \mathrm{CH}_{2} \mathrm{CH}_{2} \mathrm{CH}_{2}\right)$.

\section{2-(4-Trifluoromethylphenyl)-piperidine}

Yellow oil, $(130 \mathrm{mg}, 98 \%), \delta_{\mathrm{H}}\left(300.13 \mathrm{MHz}, \mathrm{CDCl}_{3}\right) 7.55(2 \mathrm{H}, \mathrm{d}, J$ 8.1, aryl H), $7.48(2 \mathrm{H}, \mathrm{d}, J$ 8.1, aryl $\mathrm{H}), 3.61-3.68(1 \mathrm{H}, \mathrm{m}, \mathrm{NHCH}), 3.27-3.32\left(1 \mathrm{H}, \mathrm{m}, \mathrm{NHCH}_{\mathrm{a}} \mathrm{CH}_{\mathrm{b}}\right), 2.76-2.84\left(1 \mathrm{H}, \mathrm{m}, \mathrm{NHCH}_{\mathrm{a}} \mathrm{CH}_{\mathrm{b}}\right)$, 1.95-1.42 (7H, m, $\left.\mathrm{NHCH}_{2} \mathrm{CH}_{2} \mathrm{CH}_{2}\right)$.

\section{2-Thiophenyl-2-yl-piperidine}

Yellow oil $(40 \mathrm{mg}, 20 \%), \delta_{\mathrm{H}}\left(300.13 \mathrm{MHz}, \mathrm{CDCl}_{3}\right)$ 7.16-7.12 $(1 \mathrm{H}, \mathrm{m}, \mathrm{SCH}), 6.89-6.93(2 \mathrm{H}, \mathrm{m}$, $\mathrm{SCHCHCH}), 3.85-3.91(1 \mathrm{H}, \mathrm{m}, \mathrm{NHCH}), 3.10-3.17\left(1 \mathrm{H}, \mathrm{m}, \mathrm{NHCH}_{\mathrm{a}} \mathrm{CH}_{\mathrm{b}}\right), 2.71-2.79(1 \mathrm{H}, \mathrm{m}$, $\left.\mathrm{NHCH}_{\mathrm{a}} \mathrm{CH}_{\mathrm{b}}\right), 1.82-2.96\left(3 \mathrm{H}, \mathrm{m}, \mathrm{NH}+\mathrm{CH}_{2}\right) 1.42-1.63\left(4 \mathrm{H}, \mathrm{m}, 2 \times \mathrm{CH}_{2}\right)$.

\section{2-phenyl-cycloheptylamine}

Colourless oil, $(24 \mathrm{mg}, 12 \%), \delta_{\mathrm{H}}\left(300.13 \mathrm{MHz}, \mathrm{CDCl}_{3}\right) 7.25(5 \mathrm{H}, \mathrm{m}$, aryl H), $3.75(1 \mathrm{H}, \mathrm{dd}, J$ 2.2, 10.1, $\mathrm{NHCH}), 3.12\left(1 \mathrm{H}, \mathrm{dt}, J 15.3,3.6, \mathrm{NHCH}_{\mathrm{a}} \mathrm{H}_{\mathrm{b}}\right), 2.86\left(1 \mathrm{H}, \mathrm{m}, \mathrm{NHCH}_{\mathrm{a}} H_{\mathrm{b}}\right), 1.95(1 \mathrm{H}, \mathrm{m}, \mathrm{CH}), 1.71$ $(7 \mathrm{H}, \mathrm{m}, 7 \mathrm{xCH})$.

\section{Asymmetric reduction of 9 to $10 .^{6}$}
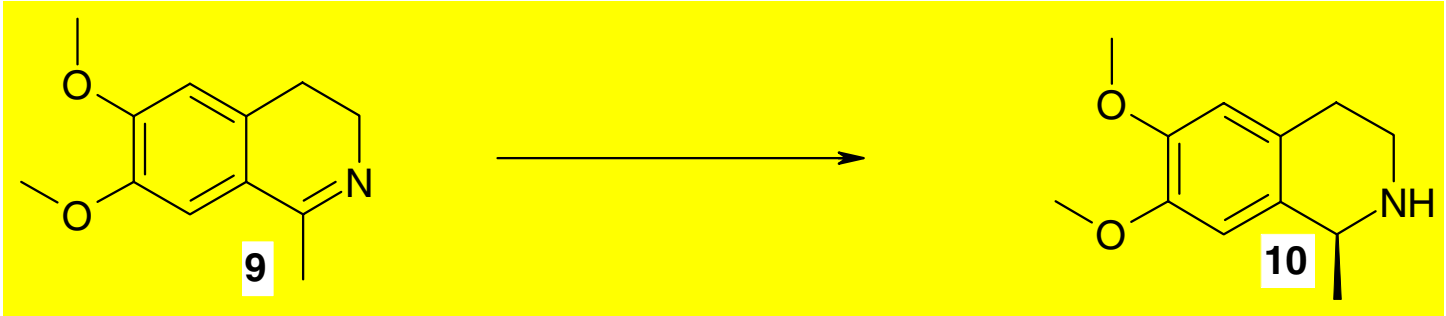

A mixture of (p-cymene) ruthenium (II) chloride dimer $(2.3 \mathrm{mg}, 0.0037 \mathrm{mmol})$ and (1R,2R)-TsDPEN $(2.7 \mathrm{mg}, 0.0073 \mathrm{mmol})$ in a $5: 2$ formic acid-triethylamine mixture $(4 \mathrm{~mL})$ was stirred at $28^{\circ} \mathrm{C}$ for 30 min. A solution of imine (300 $\mathrm{mg}, 1.5 \mathrm{mmol})$ in anhydrous acetonitrile $(2 \mathrm{ml})$ was added and the solution was stirred for $16 \mathrm{~h}$. The solution was poured onto saturated $\mathrm{Na}_{2} \mathrm{CO}_{3}$ solution $(10 \mathrm{~mL})$ and extracted with DCM (3 x $20 \mathrm{~mL})$. Combined organics were washed with brine $(10 \mathrm{~mL})$ and dried $\left(\mathrm{MgSO}_{4}\right)$, the solvent was removed under reduced pressure and the residue purified by flash column chromatography (50-100 \% v/v ethyl acetate-hexanes gradient elution) to afford (S)-(-)-6,7dimethoxy-1-methyl-1,2,3,4-tetrahydroisoquinoline $\mathbf{1 0}^{5}$ as a pale orange oil which crystallised on 
standing (296 mg, $95 \%) ; \quad-52.0\left(c 1 \mathrm{~g} / 100 \mathrm{~mL}, \mathrm{CHCl}_{3}\right) ; v_{\max }$ (neat) $/ \mathrm{cm}^{-1} 3270,2929,1511,1452$, 1252, 1220, 1114, 784; $\delta_{\mathrm{H}}\left(300.13 \mathrm{MHz}, \mathrm{CDCl}_{3} ;\left(\mathrm{CH}_{3}\right)_{4} \mathrm{Si}\right) 6.61(1 \mathrm{H}, \mathrm{s}, \mathrm{Ph} \mathrm{H}), 6.56(1 \mathrm{H}, \mathrm{s}, \mathrm{Ph} \mathrm{H})$, $4.06\left(1 \mathrm{H}, \mathrm{q}, \mathrm{J}\right.$ 6.7, $\left.\mathrm{CH}_{3} \mathrm{CH}\right), 3.85\left(3 \mathrm{H}, \mathrm{s}, \mathrm{OCH}_{3}\right), 3.22-3.33\left(1 \mathrm{H}, \mathrm{m}, \mathrm{CH}_{2}\right), 2.97-3.07\left(1 \mathrm{H}, \mathrm{m}, \mathrm{CH}_{2}\right)$, 2.76-2.85 (1H, m, $\left.\mathrm{CH}_{2}\right), 2.62-2.67\left(1 \mathrm{H}, \mathrm{m}, \mathrm{CH}_{2}\right), 1.46\left(3 \mathrm{H}, \mathrm{d}, \mathrm{J}\right.$ 6.4, $\left.\mathrm{CHCH}_{3}\right) ; \delta_{\mathrm{C}}\left(75.5 \mathrm{MHz}, \mathrm{CDCl}_{3}\right)$ $147.7\left(\mathrm{C}_{\mathrm{q}}\right), 147.6\left(\mathrm{C}_{\mathrm{q}}\right), 132.9\left(\mathrm{C}_{\mathrm{q}}\right), 127.3\left(\mathrm{C}_{\mathrm{q}}\right), 112.2(\mathrm{CH}), 109.5(\mathrm{CH}), 56.4\left(\mathrm{CH}_{3}\right), 56.3\left(\mathrm{CH}_{3}\right), 51.7$ (CH), $42.3\left(\mathrm{CH}_{2}\right), 30.0\left(\mathrm{CH}_{2}\right), 23.3\left(\mathrm{CH}_{2}\right) ; \mathrm{MS} \mathrm{m} / \mathrm{z}(\mathrm{EI}) 207\left(\mathrm{M}^{+}, 15\right), 206$ (20), 193 (30), 192 (100); HRMS: calc. $\mathrm{C}_{12} \mathrm{H}_{17} \mathrm{NO}_{2}$ calc. 207.1259 , found 207.1255 . The ee was determined to be $88 \%$ by derivatisation of (S)-(-)-6,7-dimethoxy-1-methyl-1,2,3,4-tetrahydroisoquinoline with 5(R)-methyl-1(chloromethyl)-2-pyrrolidinone (8) and the integration of the doublets at $1.33 \mathrm{ppm}$ and $1.40 \mathrm{ppm}$ in the ${ }^{1} \mathrm{H}$ NMR were used to calculate ee $\%$.

\section{Preparation of 11 from lactam 6,7-dimethoxy-3,4-dihydro-2H-isoquinolin-1-one.}

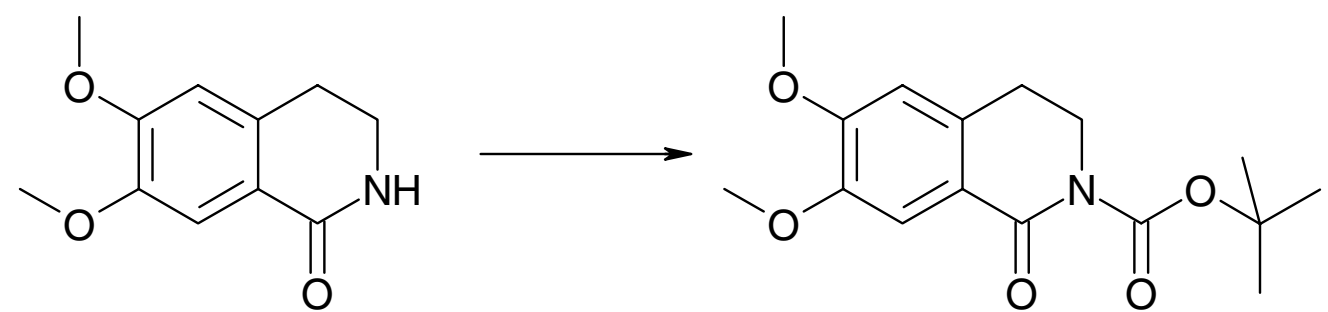

To a suspension of hexane washed $\mathrm{NaH}(0.82 \mathrm{~g}, 60 \%$ suspension in oil, $0.020 \mathrm{~mol})$ in anhydrous THF $(30 \mathrm{~mL})$ at $0^{\circ} \mathrm{C}$ was added drop wise a solution of 6,7-dimethoxy-3,4-dihydro-2H-isoquinolin-1-one $(3.92 \mathrm{~g}, 0.19 \mathrm{~mol})$, the resulting suspension was stirred at $0^{\circ} \mathrm{C}$ for $1 \mathrm{hr}$. $\mathrm{Boc}_{2} \mathrm{O}(4.05 \mathrm{~g}, 0.19 \mathrm{~mol})$ was added in 4 portions over $30 \mathrm{~min}$, a thick suspension was formed so THF (20 mL) was added and the reaction allowed to warm to $\mathrm{rt}$ and stirred for $4 \mathrm{hr}$ until complete by TLC. Water $(20 \mathrm{~mL})$ was added cautiously and the phases were separated. The aqueous layer was extracted with $\mathrm{Et}_{2} \mathrm{O}(2 \times 50 \mathrm{~mL})$ and the organics combined, dried $\left(\mathrm{Na}_{2} \mathrm{SO}_{4}\right)$, filtered and the solvent removed under reduced pressure. Residual DMF was removed azeotropically with EtOAc/Heptane (1:9) under reduced pressure to afford 6,7-dimethoxy-1-oxo-3,4-dihydro-1H-isoquinoline-2-carboxylic acid tert-butyl ester as an off white solid (4.95 g, 86\%); mp 116-119 ${ }^{\circ}$; $v_{\max }\left(\right.$ neat) $/ \mathrm{cm}^{-1} 2976,2360,2342,1749,1661,1155$; $\delta_{\mathrm{H}}\left(300.13 \mathrm{MHz}, \mathrm{CDCl}_{3} ;\left(\mathrm{CH}_{3}\right)_{4} \mathrm{Si}\right) 7.64(1 \mathrm{H}, \mathrm{s}$, aryl H), $6.65(1 \mathrm{H}, \mathrm{s}$, aryl H), $3.98(2 \mathrm{H}, \mathrm{t}, J$ 6.2, $\left.\mathrm{NCH}_{2} \mathrm{CH}_{2}\right), 3.93\left(3 \mathrm{H}, \mathrm{s}, \mathrm{OCH}_{3}\right), 3.91\left(3 \mathrm{H}, \mathrm{s}, \mathrm{OCH}_{3}\right), 2.95\left(2 \mathrm{H}, \mathrm{t}, J 6.2, \mathrm{NCH}_{2} \mathrm{CH}_{2}\right), 1.60(9 \mathrm{H}, \mathrm{s}, t$ $\mathrm{Bu}) ; \delta_{\mathrm{C}}\left(75.5 \mathrm{MHz}, \mathrm{CDCl}_{3}\right) 164.3\left(\mathrm{C}_{\mathrm{q}}\right), 153.8\left(\mathrm{C}_{\mathrm{q}}\right), 153.3\left(\mathrm{C}_{\mathrm{q}}\right), 148.5\left(\mathrm{C}_{\mathrm{q}}\right), 134.3\left(\mathrm{C}_{\mathrm{q}}\right), 122.1\left(\mathrm{C}_{\mathrm{q}}\right)$, 111.6 (CH), $109.5(\mathrm{CH}), 83.4\left(\mathrm{C}_{\mathrm{q}}\right), 56.5\left(\mathrm{CH}_{3}\right), 56.4\left(\mathrm{CH}_{3}\right), 45.1\left(\mathrm{CH}_{2}\right), 28.5\left(\mathrm{CH}_{3}\right), 28.4(\mathrm{CH})$; $\mathrm{MS}$ 
m/z (CI) 307 (M+, 80), 207 (100), 178 (40), 150 (30); HRMS: calc. $\mathrm{C}_{16} \mathrm{H}_{21} \mathrm{NO}_{5}$ calc. 307.1420, found 307.1407; Anal. calc. for $\mathrm{C}_{16} \mathrm{H}_{21} \mathrm{NO}_{5}$ : C, 62.53; H, 6.89; N, 4.56, found C, 62.47; H, 6.82; N, 4.49.

\section{Preparation of $11 .^{3}$}

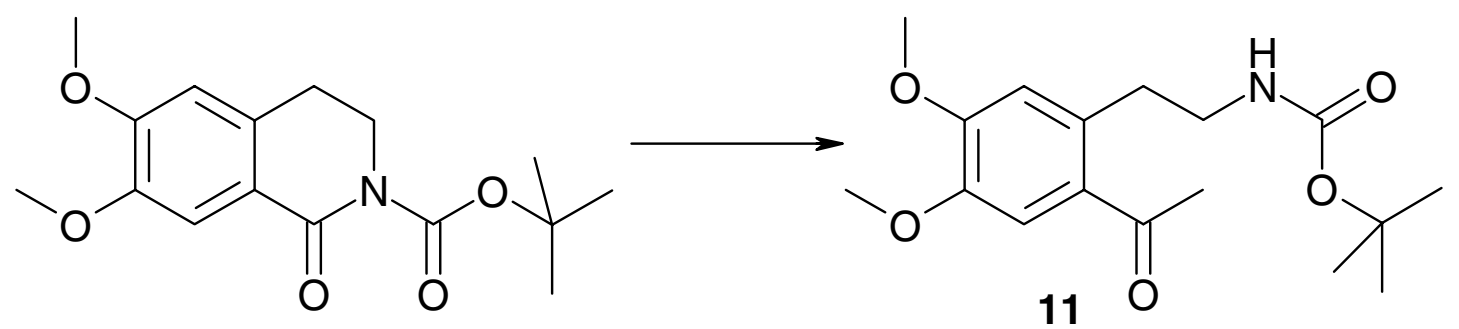

To a stirred solution of 6,7-dimethoxy-1-oxo-3,4-dihydro-1H-isoquinoline-2-carboxylic acid tertbutyl ester $(1 \mathrm{~g}, 3.3 \mathrm{mmol})$ in $\mathrm{THF}(20 \mathrm{~mL})$ at $-78^{\circ} \mathrm{C}$ was added drop wise a solution of methyllithium $(0.086 \mathrm{~g}, 1.6 \mathrm{~mol}$ in THF) over $1 \mathrm{hr} .2 \mathrm{~N} \mathrm{HCl}(10 \mathrm{~mL})$ was added and the reaction warmed to rt. The layers were separated and the aqueous layer extracted with DCM (2 x $20 \mathrm{~mL})$, the organics were combined, dried $\left(\mathrm{Na}_{2} \mathrm{SO}_{4}\right)$, filtered and the solvent removed under reduced pressure. The crude oil was purified by flash column chromatography $(0-15 \% \mathrm{v} / \mathrm{v}$ ethyl acetate-hexanes gradient elution) to afford [2-(2-acetyl-4,5-dimethoxy-phenyl)-ethyl]-carbamic acid tert-butyl ester $(11)^{3}$ as a pale yellow powder $(0.5 \mathrm{~g}, 47 \%) ; \mathrm{mp} 107-110^{\circ} \mathrm{C} ; v_{\max }($ neat $) / \mathrm{cm}^{-1} 3312,2969,2936,1680,1539,1147 ; \delta_{\mathrm{H}}(300.13$ $\left.\mathrm{MHz}, \mathrm{CDCl}_{3} ;\left(\mathrm{CH}_{3}\right)_{4} \mathrm{Si}\right) 7.21(1 \mathrm{H}, \mathrm{s}$, aryl H), $6.73(1 \mathrm{H}, \mathrm{s}$, aryl H), $5.03(1 \mathrm{H}, \mathrm{bs}, \mathrm{NH}), 3.92(6 \mathrm{H}, \mathrm{s}, 2 \mathrm{x}$ $\left.\mathrm{OCH}_{3}\right), 3.37\left(2 \mathrm{H}, \mathrm{m}, \mathrm{NHCH}_{2}\right), 3.02\left(2 \mathrm{H}, \mathrm{t}, J 7.0, \mathrm{NHCH}_{2} \mathrm{CH}_{2}\right), 2.58\left(3 \mathrm{H}, \mathrm{s}, \mathrm{COCH}_{3}\right), 1.41(9 \mathrm{H}, \mathrm{s}, t-$ $\mathrm{Bu}) ; \delta_{\mathrm{C}}\left(75.5 \mathrm{MHz}, \mathrm{CDCl}_{3}\right) 156.5\left(\mathrm{C}_{\mathrm{q}}\right), 152.1\left(\mathrm{C}_{\mathrm{q}}\right), 147.0\left(\mathrm{C}_{\mathrm{q}}\right), 134.9\left(\mathrm{C}_{\mathrm{q}}\right), 129.9\left(\mathrm{C}_{\mathrm{q}}\right), 114.7(\mathrm{CH})$, 113.6 (CH), $79.3\left(\mathrm{C}_{\mathrm{q}}\right), 56.6\left(\mathrm{CH}_{3}\right), 56.4\left(\mathrm{CH}_{3}\right), 42.5\left(\mathrm{CH}_{2}\right), 34.4\left(\mathrm{CH}_{2}\right), 29.8\left(\mathrm{CH}_{3}\right), 28.8\left(\mathrm{CH}_{3}\right) ; \mathrm{MS}$ m/z (EI) $323\left(\mathrm{M}^{+}\right.$, 4), 206 (80), 205 (100), 57 (25); HRMS: calc. $\mathrm{C}_{17} \mathrm{H}_{25} \mathrm{NO}_{5}$ calc. 323.1734, found 323.1729; Anal. calc. for $\mathrm{C}_{17} \mathrm{H}_{25} \mathrm{NO}_{5}$ : C, 63.14; H, 7.79; N, 4.33, found C, 63.22; H, 7.77; N, 4.28.

\section{Conversion of 11 to 9 using the one-pot protocol.}

The conversion of $\mathbf{1 1}$ to $\mathbf{9}$ was carried out in $85 \%$ yield following the general procedure described above for the monocyclic compounds. The resulting product had identical ${ }^{1} \mathrm{H}$ and ${ }^{13} \mathrm{C}$ spectra to that of reduction of 9 . The ee was determined to be $88 \%$ by derivatisation of (S)-(-)-6,7-dimethoxy-1methyl-1,2,3,4-tetrahydroisoquinoline with 5(R)-methyl-1-(chloromethyl)-2-pyrrolidinone (8) and the integration of the doublets at $1.33 \mathrm{ppm}$ and $1.40 \mathrm{ppm}$ in the ${ }^{1} \mathrm{H}$ NMR were used to calculate ee\%. $[\alpha]_{\mathrm{D}}^{22}-51.7\left(c 1 \mathrm{~g} / 100 \mathrm{~mL}, \mathrm{CHCl}_{3}\right)$; 


\section{General procedure for determination of enantiomeric excess.}

To a suspension of sodium hydride $(0.13 \mathrm{mmol}$, washed with hexanes and dried under vacuum $)$ in DCM $(1 \mathrm{~mL})$ at $0^{\circ} \mathrm{C}$ was added a solution of amine $(0.12 \mathrm{mmol})$ in DCM $(0.5 \mathrm{~mL})$, the resulting suspension was stirred for $1 \mathrm{hr}$ before a solution of halide $8(0.13 \mathrm{mmol})$ in DCM $(0.25 \mathrm{~mL})$ was added and stirred for $2 \mathrm{hrs}$. Water $(1 \mathrm{~mL})$ was added cautiously and the organic layer was separated, the aqueous layer was extracted with further DCM $(2 \times 1 \mathrm{~mL})$, the organic extracts were combined, dried $\left(\mathrm{Na}_{2} \mathrm{SO}_{4}\right)$, filtered and the solvent removed under reduced pressure to afford the crude products. These were analysed without purification. The e.e. was determined from the 2 methyl doublets around 1 ppm, a $\mathrm{CH}_{2}$ signal around 3 ppm or an $\mathrm{AB}$ system around $4 \mathrm{ppm}$.

\section{References.}

1) Giovannini, A.; Savoia, D.; Umani-Ronchi, A. J.Org.Chem., 1989 54, 228-234.

2) Willoughby, C. A.; Buchwald, S. L. J.Am.Chem.Soc., 1994, 116, 1994; 8952-8965.

3) Lenz, G. R.; Woo, C.-M.; Hawkins, B. L. J.Org.Chem., 1982, 47, 3049-3053.

4) Hunt, J. C. A.; Laurent, P.; Moody, C. J. J.Chem.Soc.Perkin Trans.1, 2002, 2378 - 2389.

5) Pedrosa, R.; Andres, C.; Iglesias, J. M. J.Org.Chem. 2001, 66, 243 - 250.

6) Uematsu, N.; Fujii, A.; Hashiguchi, S.; Ikariya, T.; Noyori R., J. Am. Chem. Soc., 1996, 118, 491617. 
